\title{
Security Architectures in Constrained Environments: a Survey
}

\author{
Philippe Pittoli, Pierre David, Thomas Noël \\ \{p.pittoli,pda,noel\}@unistra.fr \\ Université de Strasbourg, Laboratoire ICube
}

\begin{abstract}
This paper is a survey of the current work to understand the available security measures in the literature for constrained environments. First of all, this paper introduces the specific problem of security in constrained environments and the need for a consistent solution. The necessary set of security measures that must be present in an architecture is reviewed. Second, an overview of such consistent solutions in the literature is provided. Finally, a comparison of these solutions is performed based on the security and constraint aspects.
\end{abstract}

Keywords: security architectures, constrained environments, IETF, OSCAR, Object Security, COSE, ACE, DCAF, OAuth, DTLS, Kerberos, MQTT-SN, IPsec, SSH, OSCOAP

\section{Introduction}

With the advance in technology, it is now possible to automate a great number of tasks such as at home or in industry with the help of sensors and actuators, a temperature meter or a light bulb for example. These are used to make everyday life more comfortable by doing repetitive tasks: warming or cooling a room at fixed hours, turning a light on when someone enters, detecting anomalies in an industrial process and even more. These technologies are based on constrained environments.

Devices and networks are qualified as constrained [6] because the devices in use may be running on batteries and they have very limited memory, computation and communication capacities. The communication technologies used on these devices, such as IEEE 802.15.4 or Bluetooth, have limited message size and low bandwidth. These limitations imply security challenges as described in [24] and [11].

Security must be taken into account in the design of a consistent security solution for constrained environments. A consistent solution must cover protection of communications (authenticity, integrity, confidentiality), authenticity verification of the peers, access control and key management system. We name such a consistent solution a security architecture.

This paper provides a high level formal definition of security architecture that permits to find the solutions in the literature that address these security concerns. Each architecture is described with its objectives, advantages and drawbacks, along with a concise summary of operations (including the involved protocols and information exchanged) as described in the definition. Then we compare architectures on several aspects (number of required interactions, packet sizes, information stored and exchanged, authentication methods, required algorithms, and so on). This comparison follows scenarios showing different requirements (in authentication and key management) in a constrained environment context. Finally, from this comparison and according to the constraints that a deployment may have, we determine the relevant architecture.

Scope of the survey. First of all, this document focuses on link layer independent security architectures, to let network operators choose the communication medium depending on the situation.

Second, this document provides a focus on the operational phase of an architecture. There are several phases for a sensor network: the initial bootstrap when the security informations (e.g.: shared keys and certificates) are installed into the devices, the hardware deployment at the final location, the potential association, acceptance or even election between nodes, the operational phase when the architecture achieves its main purpose and finally the removal of a node which reaches its end-of-life. These phases will vary according to the purposes of the architecture, the deployment 
environment, the characteristics of the devices, the number of nodes, and so on. As a result, documents describing architectures in the literature are focusing mainly on the operational phase, and other phases are considered out of scope; this is also the case for this document.

Section 2 provides a definition of security architectures based on observation of current deployments. Section 3 provides an overview of the main existing architectures according to our definition, described with a common language. Section 4 provides a comparison of these architectures based on security aspects and constraints of the underlying networks. Section 5 provides an overview of the relevance of each architecture according to deployment constraints. Section 6 provides the related work, and Section 7 concludes this paper.

\section{Definition of security architecture}

Scientific communities, companies and standardization organisations described different architectures. In this section, we define a common language to describe and compare these architectures. We first provide a background of constrained environments to find the common security needs for a consistent security solution. Then we give a definition of security architecture, which leads to a practical figure to represent an architecture.

\subsection{Context}

Sensors and actuators are located on devices with communication capabilities, letting them be controlled remotely. For cost and deployment reasons, these devices are often very cheap and have low capabilities in memory, computation, communication and may also have limited access to a power source.

The following services (software) and principles form a security architecture.

The resource server is running on a physical device with attached sensors and/or actuators, providing them communication capabilities. The resource server holds sensitive data (from sensors) and may have actions in the physical world.

The client may be located on a powerful device (such as a smartphone) or a constrained one. Multiple clients can coexist, each of them may have different rights and access different resources.

The authentication service verifies one or many attributes of a client (for example its identity). The authorization service verifies allowed rights and eventually provides authorization grants to the client.

\subsection{Discussion on basic security features}

Authentication and authorization should be mutual. The authentication service should authenticate the client to learn its identity (the client must prove it has the right secret or private keying material), and the client's rights should be verified by the authorization service. Similarly, the client should authenticate every entity it interacts with, to prevent unauthorized disclosure of private information. The client should also verify that the resource server is authorized to perform such operation, again, to prevent unauthorized disclosure.

For authorization, on one hand, some deployments only require coarse grained authorizations where an authenticated client has access to everything. This may be the case for instance in home automation where a single remote can control every part of the house. On the other hand, in several cases, deployments require fine-grained authorizations where there are multiple clients and the server domain principal wants to grant as little rights to each client as possible. For example, a transport company has a set of containers, each one of them can be located with a GPS system and has a door lock and a remote temperature regulation in order to preserve the transported material. The company wants to let a customer access the location of the merchandise and may change the fan regulation, but only the company's employees loading and unloading the containers would be able to open the door.

Finally, communications (basic packet transmission) must be protected. Both sides of the communication should be able to tell if a received packet was modified (integrity) or if it comes from the right place (authenticity). Both sides must be ensured that only the peers understand the transmission (confidentiality) ${ }_{1}^{1}$. A replay protection should additionally be provided, a passive eavesdropper should not be able to perform an authentication replaying past messages.

\footnotetext{
${ }^{1}$ Confidentiality may not be required in some cases, or more specifically informations must be revealed to authorized parties, which is named "visibility". For the sake of simplicity, we focus on confidentiality only.
} 


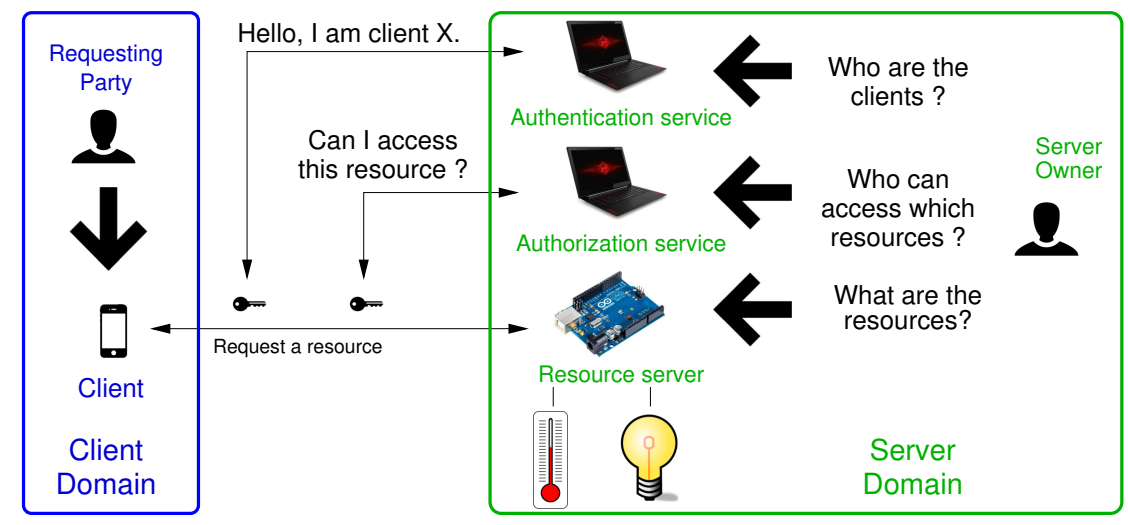

\begin{tabular}{|lll|}
\hline Legend & Gessage exchange \\
Authority & Blue: & Server domain \\
& & Configure \\
\hline
\end{tabular}

Figure 1: Example of components in a security architecture.

To summarize, architectures considered in this document are already bootstrapped, deployment and initial configurations are done and the focus is on the operational phase. In those architectures there is a client wanting to access a resource provided by a resource server. The server domain must authenticate the client and authorize to access the resource. Authentication, authorization and requesting steps are done in many ways following the considered architecture and this document compares the different ways to access such a resource. Cryptographic protection against eavesdroppers and man-in-the-middle attacks should be provided.

Another way to say is: a consistent solution to secure the communication of an architecture must provide counter measures to passive eavesdropping and active attacks, such as man-in-the-middle attacks involving message alteration or packet forgery. Any architecture that complies with these requirements is considered a security architecture.

Next section provides a formal definition in order to find architectures in the literature, describe them with a common language and, for each one of them, to give a concise summary.

\subsection{Definition}

We define a security architecture as a 4-tuple $\langle E, P, D, R>$.

Entities (E) are the components (software) providing functionalities in the architecture. These are controlled by some principals (P) which are persons or organizations. Security domains (D) represent the link between the principals and the set of entities they control. Finally, relations (R) are the interactions between the entities.

Figure 1 shows an introduction to these concepts: a client requests a resource, but must be authenticated ("do you recognize me?") and must be authorized ("can I do this?"). To delimit the domains on the figure, the server domain principal and its entities are in green text, the requesting party and its client entity are in blue text.

\subsubsection{Entities $(\boldsymbol{E})$}

Entities represent the software deployed on a device providing a service in the architecture. For example:

- the client of the resource;

- the resource server providing resource representations from the sensors and activating actuators;

- the client authentication service ensures that only a known client can access the server domain;

- the resource access authorization server which grants access to a resource to a set of clients.

Multiple entities may run on a single device. 


\subsubsection{Principals $(\boldsymbol{P})$}

Principals are the people or the organizations in charge of the entities, which means they control their behavior and policy. For example, the server domain principal is in charge of the authentication and authorization services to access its resource service.

\subsubsection{Security domains $(D)$}

Security domains represent the set of entities controlled by a principal. Each domain has its own mechanisms, security policy, key management and interactions between entities. A domain controlled by a principal $\mathrm{p}$ is defined as $\operatorname{Dom}(p)=\{e \in E \mid p$ controls $e\}$. Consequently, D is defined as:

$$
D=\{\operatorname{Dom}(p) \mid p \in P\}
$$

Except when an architecture explicitly defines specific domains, we will assume that there is a server domain principal which controls all entities except the client. For example, a company allows computers (clients) from another organization (other domain) to use their printer (resource server) once authenticated on a company's server (authentication and authorization entities).

\subsubsection{Relations $(\boldsymbol{R})$}

Relations describe interactions between entities in the architecture. A relation is a tuple $\left\langle e_{1}\right.$ op $\left.e_{2}, l, o, i\right\rangle$ where $e_{1} \in E, e_{2} \in E$, op $\in\{\rightarrow, \leftrightarrow\}, l \subseteq L, o \subseteq O, i \subseteq I$.

- $\mathrm{E}$ is the set of entities previously detailed;

- op represents information direction between two entities. The operation $\leftrightarrow$ is used to indicate a bidirectional message exchange where the sequence of messages is not yet known or to simplify relations for readability reasons, to group messages for a DTLS connection for instance.

- $\mathrm{L}$ is the set of all protocols used in this architecture, each relation uses a subset $l$ of these protocols.

- $\mathrm{O}$ is the set of all properties, each relation has a subset $o$ of these properties. There is a great number of communication properties, this document is focusing on the following set to describe inherent protocol properties of relations:

- reliability F;

- order O;

- confidentiality C, mutual authentication MA, integrity I;

- availability A (denial of service protection);

- anti-replay protection $\mathrm{R}$;

- accountability AC.

- I is the set of all informations exchanged in this architecture. The subset $i$ represents the pieces of information exchanged in a relation, for example to mutually authenticate both sides of the communication, to negotiate the algorithms or to perform the request.

$\mathrm{R}$ is thus the set of all relations which characterizes the implementation of an architecture.

For example, a client requesting a resource on a server RS with CoAP only could be represented as the relations in Table 1 


\begin{tabular}{|l|c|c|c|c|}
\hline$\#$ & entities & protocols & properties & informations \\
\hline $\mathrm{R}_{1}$ & $\mathrm{C} \rightarrow \mathrm{RS}$ & $\mathrm{CoAP}$ & $\mathrm{F}$ & URI \\
\hline $\mathrm{R}_{2}$ & $\mathrm{RS} \rightarrow \mathrm{C}$ & $\mathrm{CoAP}$ & $\mathrm{F}$ & value \\
\hline
\end{tabular}

Table 1: Relation examples.

\subsection{Discussion on minimal security properties}

There is a minimal set of properties required in an architecture to protect communications, data and behavior in order to be considered secured.

First, a security architecture must provide data protection, which means integrity verification $I$, authenticity verification $M A$ and confidentiality $C$ of the data during the transfer between two entities. Replay protection $R$ is also necessary for communications, in order to prevent eavesdroppers from replaying old messages. Data protection involves a channel between the two sides of a communication and cryptography to encrypt, to create message integrity and message authentication codes. Another way to protect data is to use an object security, which means to secure only the data (message payload) through cryptography (signature, encryption, hashing). This helps to reduce the number of messages, to facilitate group communications (via multicast for example), to easily cache messages and still perform end-to-end security: intermediaries such as proxies and cache services do not need the deciphered value to accomplish their task, they can also verify the authorship of the message when it is signed, etc. Other optional mechanisms may be employed, such as availability protection $A$ to prevent attackers from overloading the memory of devices with half-open connections for instance, along with data origin to know the resource server originating the data. Each relation has a set of these properties. Non security oriented properties such as reliability $F$ and order $O$ may be required or induced by the security layer.

Second, mutual authentication between the client and an authentication entity must be performed. Authentication can be performed in many ways, using by example one or more of:

- a password or a pre-shared key (PSK), used for example to derive the cryptographic material for a communication or to directly encrypt the resource representation;

- two pairs of public and private keys (one pair on each side), with public keys shared on both sides;

- certificates (provided by a trusted or verifiable third party), used in combination of the public and private keys from the previous option, to limit the validity of the keys in time and identify a peer via a trusted third party;

- tickets, short term certified authentication and authorization representations, provided by a trusted entity.

Other authentication mechanisms exist with a different trust model such as PGP and the web of trust [8], but are out of the scope of this paper. Authentication may involve asymmetric algorithms (as in public key cryptography) associated with the use of certificates or raw public keys and implies heavy computations even with elliptic curves optimisations [26]. Pre-shared keys (and tickets) require only symmetric algorithms which are far more efficient and should be preferred when possible in constrained environments.

Third, authorization must be granted to the client before the request is processed by the resource server. Access control can be divided into two simple categories: implicit and explicit. Implicit access control is a coarse grained control over the resources: it does not provide a link between a resource and a client, any client (or authenticated client) has access to all resources in the domain without further requirements. Explicit access control means that in the architecture, authorizations are foreseen and link a set of resources to a set of clients, allowing as precise control over resources as one may need. The architecture may use authorization grants to inform the client of its rights (which operation to perform on which resource server). For example, those rules will discriminate a client or a connection on any available element, such as the client id or the time of the connection, then the client will have access to a set of resources, resource servers or resource types on different resource servers.

Finally, key management refers to the establishment of cryptographic keying material used to secure the communication between two entities. Key management is considered automatic when a new short-term cryptographic key is generated for a session, derived from a long-term key. Best current practices about key management are discussed in [4]. 
Other mechanisms may be employed to strengthen the protection or to facilitate the deployment. For example, packet size concealing and encrypted dummy packets hide real network usage, cryptographic agility allows to choose cryptographic algorithms on-the-fly at the connection and avoids to change protocol for a cryptographic update, resource discovery to explore available resources or to handle dynamic resources and so on.

Authentication mechanisms are dependent of the situation: in a LAN with devices controlled by a single entity, using pre-configured keys as authentication mechanism may be realistic. If there are numerous clients, certificates with limited validity period can be a better choice: the resource server performing the authentication will not have to keep a state for every client. If certificates have limited validity period, the resource server has to check the expiration date of certificates (a real-time clock is therefore needed) and the signature (a public key and consequently asymmetric cryptography are needed).

\subsection{Security architecture abstract example}

Figure 2 presents a security architecture example according to our definition. It involves two principals, the requesting party and the server domain principal, and a minimal set of entities: the client, the resource server, the authentication and authorization services. Domains are the client domain and the server domain (with the resource server, the authentication and authorization services). Several clients (in different domains) can request a resource in the server domain. The figure shows two domains:

$$
\operatorname{Dom}(\text { client })=\{\text { Client }\}
$$

Dom $($ server $)=\{$ Authentication, Authorization, $R S\}$

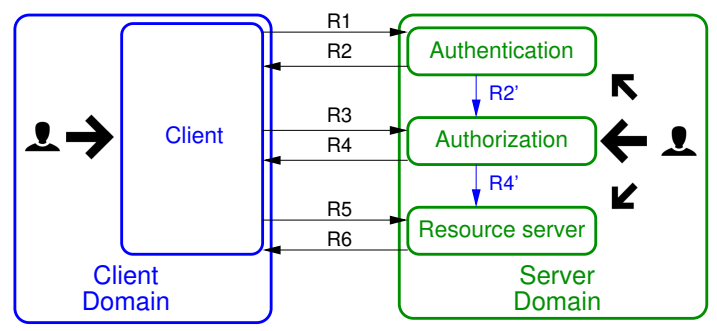

Figure 2: Security architecture example.

The following relations (table 2) show the exchanges between the entities ${ }^{2}$, and how mechanisms such as mutual authentication and authorization are performed. More specifically, the key $k_{C}$ is used by the different entities without messages to agree on it, so the authentication is based on this pre-shared key. In this example, the authorization mechanism is explicit and messages are exchanged to grant a permission. The protocol used is Constrained Application Protocol (CoAP) [46] only. No architecture should have requirements on physical and link layers, we focus on architectures which are independent from these layers and adaptable to any communication technology equivalent to IEEE 802.15.4 or less constrained. Our example architecture does not have security properties in its relations since everything is achieved at the application layer, there is no secure tunnel at a lower layer.

Encryption is presented in the relations as: [unencrypted data] $]_{\text {ey }}$. We use this representation in relations where a lower layer does not encrypt the entire application layer, otherwise we use the "C" property.

Some relations in the architectures are not specified (as indicated in figures with blue arrows) and no assumption is made on the exchanged data, the protocol nor the communication properties. Unspecified relations may represent internal communications on a device or out of scope communications. In our example architecture, $R_{2}^{\prime}$ and $R_{4}^{\prime}$ are two

\footnotetext{
${ }^{2}$ Entities will have an abbreviated name in relations: $A N$ for AutheNtication service, $A Z$ for AuthoriZation service, $R S$ for Resource Server, $C$ for Client. In the case authentication or authorization entities are also present in the client domain, prefixes " $\mathrm{C}$ " and " $\mathrm{S}$ " are used such as $C A N$ and $C A Z$ for the client authentication and authorization entities (respectively $S A N$ and $S A Z$ for the server domain).
} 
examples of such unspecified relations. These relations $\left(R_{2}^{\prime}\right.$ and $\left.R_{4}^{\prime}\right)$ could represent in this example the authentication service sharing a secret with the authorization service (relation $R_{2}^{\prime}$ ) then with the resource server (relation $R_{4}^{\prime}$ ).

Following the relations in this example, protocols used are $\mathrm{L}=\{\mathrm{CoAP}\}$, since CoAP is the only protocol used. Properties $\mathrm{O}=\{\mathrm{F}\}$, this architecture only needs reliability $(\mathrm{F})$ to work properly, which can be achieved at the application layer with CoAP (with confirmable/acknowledgement messages) or required at the link layer. Shared informations $\mathrm{I}=$ \{client id, ticket, ticket', request, response $\}$ where the client id is an arbitrary string, tickets content is explicitly detailed in the relations, and finally request and response are related to CoAP.

Note that $\perp$ in relations represents the unknown set of values.

\begin{tabular}{|c|c|c|c|c|}
\hline$\#$ & entities & protocols & properties & informations \\
\hline $\mathrm{R}_{1}$ & $\mathrm{C} \rightarrow \mathrm{AN}$ & CoAP & $\bar{F}$ & $\begin{array}{l}\text { client id } \\
\text { The client sends its identity to the authentication entity. CoAP is used in } \\
\text { this exchange and the communication is reliable (by CoAP or the application layer) }\end{array}$ \\
\hline $\mathrm{R}_{2}$ & $\mathrm{AN} \rightarrow \mathrm{C}$ & CoAP & $\mathrm{F}$ & $\begin{array}{c}{[\text { ticket }]_{\mathrm{k}_{\mathrm{C}}}} \\
\text { Ticket }=\{\text { timestamp, AN signature }\}\end{array}$ \\
\hline $\mathrm{R}_{2}^{\prime}$ & $\mathrm{AN} \rightarrow \mathrm{AZ}$ & $\perp$ & $\perp$ & $\stackrel{\perp}{\perp}$ This relation is not specified, no assumption is made \\
\hline $\mathrm{R}_{3}$ & $\mathrm{C} \rightarrow \mathrm{AZ}$ & CoAP & $\mathrm{F}$ & $\begin{array}{c}{[\text { ticket, request }]_{\mathrm{k}_{\mathrm{C}}}} \\
\text { The request is a resource and an action URI }\end{array}$ \\
\hline $\mathrm{R}_{4}$ & $\mathrm{AZ} \rightarrow \mathrm{C}$ & CoAP & $\mathrm{F}$ & $\begin{array}{c}{[\text { ticket', request }]_{\mathrm{k}_{\mathrm{C}}}} \\
\text { Ticket' }=\{\text { timestamp, AZ signature }\}\end{array}$ \\
\hline $\mathrm{R}_{4}^{\prime}$ & $\mathrm{AZ} \rightarrow \mathrm{RS}$ & $\perp$ & $\perp$ & $\perp$ \\
\hline $\mathrm{R}_{5}$ & $\mathrm{C} \rightarrow \mathrm{RS}$ & CoAP & $\mathrm{F}$ & {$[\text { ticket', request }]_{\mathrm{k}_{\mathrm{C}}}$} \\
\hline $\mathrm{R}_{6}$ & $\mathrm{RS} \rightarrow \mathrm{C}$ & CoAP & $\mathrm{F}$ & {$[\text { response }]_{\mathrm{k}_{\mathrm{C}}}$} \\
\hline
\end{tabular}

Table 2: Relations for our example architecture.

In more complex architectures, secure connections between two entities will have abbreviated relations for readability reasons (as indicated in figures with magenta links).

In the following architectures, the requesting party and the server domain principal are omitted from the figures since they always take care of their respective domain.

\section{Architectures under scrutiny}

In section 2.3 we have defined consistent security solutions designated as security architectures. This section now provides an overview of the main security architectures found in the literature according to our definition.

In order to extend this survey, we defined some simple architectures based on a single security mechanism (DTLS, IPSec, SSH and OSCOAP). These simple architectures, albeit basic, have a good likelihood to be deployed in practice, so they are interesting to compare with other architectures which are also often more complex.

For each architecture, a model is presented with its relations. Where appropriate, a severely constrained deployment possible with the architecture is also presented, when entities are deployed on separate constrained devices.

\section{1. $A C E D C A F$}

ACE DCAF (Authentication and Authorization for Constrained Environments Delegated CoAP Authorization Framework) is described in an IETF draft [10], use cases of this work are described in [41]. Its purpose is to provide an architecture for authorization in constrained environments and to let the communication between the client and the resource server be authorized by both sides, the requesting party and the server domain principal. The client can only exchange information with the resource server if both principals (client and server domains) agree.

To achieve its purpose, each domain has its own authentication and authorization services. This allows each domain to precisely control communications of their entities with the other domain.

In the client domain, this allows to restrict the duration of a communication between its clients and resource servers by providing short-term cryptographic material. This prevents a corrupt client from requesting resource servers indefinitely, forcing the server domain to update its cryptographic material. 
In the server domain, this allows a central management of the connections and cryptographic material on a more powerful device than resource servers when the deployment requires it.

The architecture is generic enough to be relevant in several cases, for example where there are two domains with an authentication plus authorization device to centralise identity management and access control in both domains. This architecture also takes into account simpler cases, where the client (or server) domain is only composed of a unique device.

Entities, Principals, Domains, Relations

Figure 3 shows the architecture model. Two domains are defined in this architecture:

$$
\begin{gathered}
\text { Dom }(\text { client })=\{C A N, C A Z, C\} \\
\text { Dom }(\text { server })=\{S A N, S A Z, R S\}
\end{gathered}
$$

Each domain has authentication and authorization entities, they grant authorization for the client to access the resource server. In each domain, authentication and authorization entities are linked together in the architecture: relations between those entities are not explicitly defined, they are by default assumed to be on the same device.

For the communication, the authentication and authorization services of the client's domain verify the communication following the domain security policy, then they request the other domain authentication and authorization entities. If the server domain agrees, cryptographic material with an authorization representation are sent to the client via the client domain authentication and authorization entities. Finally, a secure connection is established between the client and the resource server using DTLS with pre-shared keys.

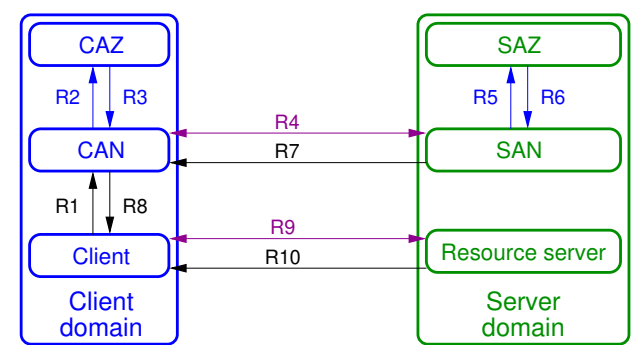

Figure 3: ACE DCAF architecture model.

Table 3 describes the relations in ACE DCAF. The final connection can be persistent (in case of multiple requests).

Figure 4 presents the most constrained instance of ACE DCAF architecture as presented in the draft: entities are on separate physical devices (except for authentication and authorization entities). The client and the resource server are deployed on constrained devices which implies communication between four devices. The client requests the client domain authentication and authorization entity to allow the communication with the resource server. If this client is expected to communicate with this resource server, the authorization is granted. Then the authorization entity itself requests the authorization entity of the server domain to permit the communication. If the permission is granted on the server domain side, then the server domain authorization entity provides the cryptographic material and authorization representation (token) to the client. Finally, the secure communication is established between the client and the resource server.

\section{Advantages and drawbacks}

The main difference between this architecture and the others is its flexibility: it allows the use of a dedicated device with authentication and authorization services, in one or both domains. A complex authentication and authorization procedures (with possibly a certificate system and heavy cryptography algorithms) could be envisaged on more appropriate devices, in each domain.

Another advantage is that the resource server and the client are only required to store a single cryptographic material to contact their respective authentication and authorization services. New short-term cryptographic material is only created when a new connection occurs, and immediately destroyed after. 


\begin{tabular}{|c|c|c|c|c|}
\hline \# & entities & protocols & properties & informations \\
\hline $\mathrm{R}_{1}$ & $\mathrm{C} \rightarrow \mathrm{CAN}$ & DTLS, CoAP & $\mathrm{C}, \mathrm{I}, \mathrm{MA}, \mathrm{R}$ & request $=\{U R I A S$, URI resource, action, timestamp $\}$ \\
\hline $\mathrm{R}_{2}$ & $\mathrm{CAN} \rightarrow \mathrm{CAZ}$ & $\perp$ & $\perp$ & $\perp$ \\
\hline $\mathrm{R}_{3}$ & $\mathrm{CAZ} \rightarrow \mathrm{CAN}$ & $\perp$ & $\perp$ & authorization \\
\hline $\mathrm{R}_{4}$ & $\mathrm{CAN} \leftrightarrow \mathrm{SAN}$ & DTLS $\vee T L S$, CoAP & $\mathrm{F}, \mathrm{O}, \mathrm{C}, \mathrm{I}, \mathrm{MA}, \mathrm{A}, \mathrm{R}, \mathrm{AC}$ & $\begin{array}{c}\text { id }_{\mathrm{CAN}}, \text { idproof }_{\mathrm{CAN}}, \mathrm{id}_{\mathrm{SAN}}, \text { idproof }_{\mathrm{SAN}}, \text { request } \\
\text { idproof } \in\{\text { certificate, PSK, raw pub keys }\} \\
\text { (D)TLS connection, abbreviated for readability reasons }\end{array}$ \\
\hline $\mathrm{R}_{5}$ & $\mathrm{SAN} \rightarrow \mathrm{SAZ}$ & $\perp$ & $\perp$ & $\perp$ \\
\hline $\mathrm{R}_{6}$ & $\mathrm{SAZ} \rightarrow \mathrm{SAN}$ & $\perp$ & $\perp$ & authorization \\
\hline $\mathrm{R}_{7}$ & $\mathrm{SAN} \rightarrow \mathrm{CAN}$ & DTLS, CoAP & $\mathrm{C}, \mathrm{I}, \mathrm{MA}, \mathrm{R}$ & ticket $=\{$ server authz info, SAN timestamp, SAN lifetime, session key $\}$ \\
\hline $\mathrm{R}_{8}$ & $\mathrm{CAN} \rightarrow \mathrm{C}$ & DTLS, CoAP & C, I, MA, R & $\begin{array}{c}\text { ticket, } \mathrm{CAN}_{\text {info }} \\
C A N_{\text {info }}=\{\text { client authz info, CAN timestamp, CAN lifetime }\}\end{array}$ \\
\hline $\mathrm{R}_{9}$ & $\mathrm{C} \leftrightarrow \mathrm{RS}$ & DTLS, CoAP & $\mathrm{F}, \mathrm{O}, \mathrm{C}, \mathrm{I}, \mathrm{MA}, \mathrm{A}, \mathrm{R}, \mathrm{AC}$ & $\begin{array}{c}\text { request, id, idproof } \\
I d=\{\text { server authz info, SAN timestamp, SAN lifetime }\}\} \\
\text { Idproof }=\{\text { session key }\} \\
\text { DTLS connection then request, abbreviated for readability reasons }\end{array}$ \\
\hline $\mathrm{R}_{10}$ & $\mathrm{RS} \rightarrow \mathrm{C}$ & DTLS, CoAP & C, I, MA, R & $\begin{array}{c}\text { response } \\
\text { Connection established, } R S \text { handles requests } \\
\text { until C disconnects or the lifetime expires }\end{array}$ \\
\hline
\end{tabular}

Table 3: ACE DCAF relations.

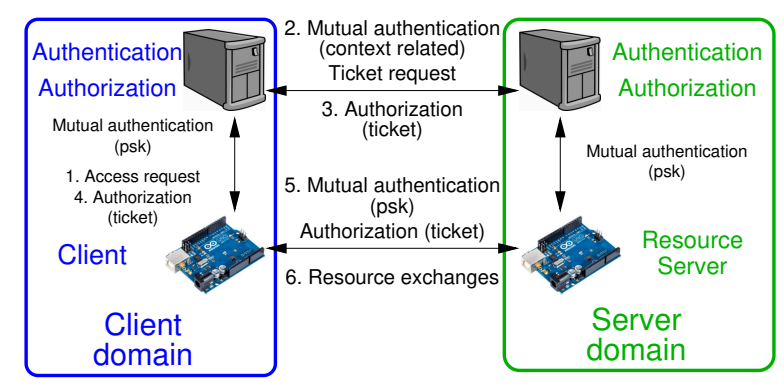

Figure 4: ACE DCAF architecture, most constrained deployment. 
Last advantage, each domain controls precisely each communication of their entities with the other domains, letting them enforce their own policy, furthermore in a centralized way. The policy involves which client contacts which resource server, the duration of the communication and the authorized requests.

As a drawback, the architecture is based on a connection protocol, requiring a connection between clients and resource servers, thus involving a few message exchanges for the handshake. This can be alleviated by using longterm connections.

\subsection{ACE OAuth}

The ACE OAuth purpose is to provide a framework for authorization in constrained environments. It is described in an IETF draft [40] from the ACE working group, like the previous architecture. The main differences are the use of the OAuth 2.0 framework and the authentication and authorization services which are only present in the server domain. This specification allows constrained environments to re-use the widespread OAuth 2.0 framework for an authorization service.

To achieve its purpose, the draft defines a framework (the main entities, their role and the main communication flow) which uses a ticket-based protocol to authenticate and authorize the client. The client gains a ticket after performing authentication and authorization, then this ticket is used by the resource server to allow access to the resource.

To validate a ticket, the resource server requires a real-time clock and may have to perform heavy cryptographic operations. If the resource server is not able to verify the ticket itself, then it is forwarded to the trusted authorization service for a confirmation of its validity.

ACE OAuth is used with a "profile": besides the main architecture, several documents show a way to use different communication protocols in the architecture, according to different deployment requirements. Currently defined profiles are OSCORE (OSCOAP) [39], DTLS [9], TLS [28], IPsec [1], EAP [27] and MQTT [44]. This paper only focuses on the OSCORE and DTLS profiles (other profiles are at early stage of writing).

Exchanges are based on the Concise Binary Object Representation (CBOR) message format [7] following the CBOR Object Signing and Encryption (COSE) [37] procedure ${ }^{3}$ DTLS may be used along with the COSE secure format, to encrypt metadata, negotiate encryption keys or to mutually authenticate $\mathrm{C}$ and $\mathrm{AN}$ for instance. ACE OAuth can also be used with EDHOC [42], an authenticated Diffie-Hellman key exchange with ephemeral keys that adds Perfect Forward Secrecy to COSE.

In this paper, we assume the use of OSCORE with pre-configured keys, an authorization server and the use of introspection (for shorter message sizes).

\section{Entities, Principals, Domains, Relations}

Figure 5 presents the ACE OAuth architecture model. There are two domains ${ }^{4}$

$$
\begin{gathered}
\operatorname{Dom}(\text { client })=\{C\} \\
\operatorname{Dom}(\text { server })=\{A N, A Z, R S\}
\end{gathered}
$$

Authentication and authorization services are linked together in the architecture.

Table 4 describes the relations in ACE OAuth. Relations $R_{6}$ and $R_{7}$ are required only if the ticket is not selfcontained. In this case, the ticket is a reference to the authorization representation in the authentication service that the resource server must request.

Informations $I$ involved in these relations are: $\mathrm{I}=\{\mathrm{id}$, id proof, token, request, response $\}$

- token: authorization representation, or a reference to in the authentication service, with a signature proving the origin;

- request, response: CoAP messages.

\footnotetext{
${ }^{3} \mathrm{COSE}$ is a set of procedures to digitally sign, encrypt and authenticate messages in the CBOR data format. Concise Binary Object Representation (CBOR) is a data format made for small code size and small message size. COSE is similar to JSON Object Signing and Encryption (JOSE, 2013) [3] but is specifically designed for constrained environments.

${ }^{4}$ In the ACE OAuth draft, the client seems to trust the authentication server, but we do not assume that they trust each other in this document. We consider that the client may be outside the server domain network, such as with other architectures.
} 


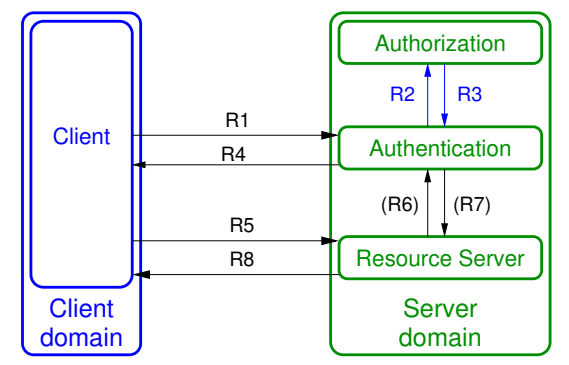

Figure 5: ACE OAuth architecture model.

\begin{tabular}{|c|c|c|c|c|}
\hline$\#$ & entities & protocols & properties & informations \\
\hline $\mathrm{R}_{1}$ & $\mathrm{C} \rightarrow \mathrm{AN}$ & CoAP & $\mathrm{C}, \mathrm{I}, \mathrm{A}, \mathrm{R}, \mathrm{AC}$ & id $_{C}$, idproof ${ }_{C}$, request \\
\hline $\mathrm{R}_{2}$ & $\mathrm{AN} \rightarrow \mathrm{AZ}$ & $\perp$ & $\perp$ & $\perp$ \\
\hline $\mathrm{R}_{3}$ & $\mathrm{AZ} \rightarrow \mathrm{AN}$ & $\perp$ & $\perp$ & authorization \\
\hline $\mathrm{R}_{4}$ & $\mathrm{AN} \rightarrow \mathrm{C}$ & CoAP & $\mathrm{C}, \mathrm{I}, \mathrm{A}, \mathrm{R}$ & id $_{\mathrm{AN}}$, idproof ${ }_{\mathrm{AN}}$, token \\
\hline $\mathrm{R}_{5}$ & $\mathrm{C} \rightarrow \mathrm{RS}$ & CoAP & $\mathrm{C}, \mathrm{I}, \mathrm{A}, \mathrm{R}$ & request, token \\
\hline$\overline{\mathrm{R}_{6}}$ & $\mathrm{RS} \rightarrow \mathrm{AN}$ & CoAP & $\mathrm{C}, \mathrm{I}, \mathrm{A}, \mathrm{R}$ & token \\
\hline $\mathrm{R}_{7}$ & $\mathrm{AN} \rightarrow \mathrm{RS}$ & CoAP & $\mathrm{C}, \mathrm{I}, \mathrm{A}, \mathrm{R}$ & $\begin{array}{c}\text { authorizations } \\
\text { Authorizations }=\text { what the client is authorized to do }\end{array}$ \\
\hline $\mathrm{R}_{8}$ & $\mathrm{RS} \rightarrow \mathrm{C}$ & CoAP & $\mathrm{C}, \mathrm{I}, \mathrm{A}, \mathrm{R}$ & response \\
\hline
\end{tabular}

Table 4: ACE OAuth relations.

\section{Advantages and drawbacks}

An advantage of this architecture is the separation of authentication and authorization entities from the resource server in the server domain, as with ACE DCAF. This implies that no heavy cryptography has to be done on the resource server for the authentication and authorization procedures, even if the client is outside the constrained network.

Another advantage is that ACE OAuth can be used only with COSE messages, requiring fewer messages than with a connection protocol such as DTLS.

As a drawback, using only COSE messages without a connection protocol implies a message size overhead. Each message, beyond carrying the request or the response, will handle authentication, algorithm and cryptographic material negotiation. However, ACE OAuth can be used along with a connection protocol to handle a negotiation and decrease the message size.

Another drawback is, unlike ACE DCAF, ACE OAuth cannot enforce a central policy in the client domain since there is no intermediate device.

\subsection{Kerberos}

Kerberos was designed in the 80's with the MIT project Athena. The last description of this architecture was made by an IETF working group in 2005 [32].

Kerberos also defines the protocol used to communicate between the entities.

Kerberos is an interesting architecture since it is the only one to explicitly dissociate the authentication, authorization and resource server entities. This allows a principal to provide authentication for different applications in the server domain or in other domains (and act as a trusted third party). Authorization is related to the final resource and can be controlled by another domain. For example, a company can allow employees from another company to access its printer.

The Kerberos purpose is to authenticate users over an insecure network. Kerberos is used in practice as an authentication mechanism for online services, such as ssh or file servers.

To achieve its purpose, Kerberos has a complex ticket-based protocol to perform authentication. An optional explicit authorization mechanism may be used if required by the service. The client requests a ticket from an au- 
thentication service, then it may ask an authorization service for a permission, and finally it contacts the resource server.

Entities, Principals, Domains, Relations

Figure 6 presents the Kerberos architecture model. There are two domains:

$$
\operatorname{Dom}(\text { client })=\{C\}
$$

$$
\operatorname{Dom}(\text { server })=\{A N, A Z, R S\}
$$

The client is authenticated by the authentication service which issues a ticket. Optionally, this ticket is used to ask for communicating with the resource server to the authorization service. Finally, the client and the resource server communicate, the client proving its identity (and authorization) with a ticket.

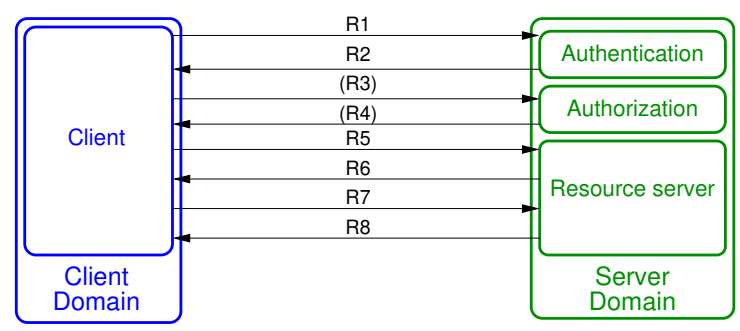

Figure 6: Kerberos architecture model.

Table 5 describes the relations in Kerberos architecture. Kerberos can be deployed on UDP or TCP transport layer, UDP is chosen in this document, so there are no reliability nor ordering properties on the relations. Kerberos defines its own message types and formats, so it is considered as a protocol and it is the only one involved in our example.

Informations $I$ involved in these relations are: $\mathrm{I}=\left\{\mathrm{AN}, \mathrm{AZ}, \mathrm{C}, \mathrm{RS}, \mathrm{n}_{1}, \mathrm{n}_{2}, \mathrm{t}_{A N}, \mathrm{t}_{A Z}, \mathrm{t}_{C}, \mathrm{t}_{C}, \mathrm{SK}, \mathrm{AK}, \mathrm{K}_{R S}, \mathrm{~K}_{A Z}\right.$, ticket granting ticket (TGT), service ticket (ST), request, response\}

- $\mathrm{n}_{1}, \mathrm{n}_{2}:$ nonces;

- AN, AZ, C, RS: identities;

- $\mathrm{t}_{A N}, \mathrm{t}_{A Z}, \mathrm{t}_{C}, \mathrm{t}_{C}=$ timestamps;

- SK, AK: session key, authentication key;

- $\mathrm{K}_{R S}, \mathrm{~K}_{A Z}$ : entity encryption keys;

- ticket granting ticket: $\mathrm{K}_{A Z}+\mathrm{C}+\mathrm{t}_{A N}$;

- service ticket: $\mathrm{K}_{R S}+\mathrm{C}+\mathrm{t}_{A Z}$;

- request, response: arbitrary.

\section{Advantages and drawbacks}

Kerberos is the only architecture to separate the authentication and the authorization entities, which allows different authorization mechanisms and set of rules for specialized services with an unique authentication mechanism and centralized identity management.

However, Kerberos messages are issued in the ASN.1 (with Basic Encoding Rules) format that is not designed for constrained environments: for example in an IEEE 802.15.4 network, message sizes are greater than the MTU (127 bytes). An example is the AS_REP which uses more than 200 bytes, even with only 10 bytes realm and domain names, thus limiting the use of Kerberos in those networks. 


\begin{tabular}{|c|c|c|c|c|}
\hline$\#$ & entities & protocols & properties & informations \\
\hline $\mathrm{R}_{1}$ & $\mathrm{C} \rightarrow \mathrm{AN}$ & Kerberos & & $\begin{array}{l}\mathrm{C}, \mathrm{AZ}, \mathrm{n}_{1} \\
\text { The client sends its ID and the ID of the authorization service it wants to reach next }\end{array}$ \\
\hline $\mathrm{R}_{2}$ & $\mathrm{AN} \rightarrow \mathrm{C}$ & Kerberos & & $\begin{array}{c}\mathrm{C}, \mathrm{TGT}_{\mathrm{K}_{\mathrm{AZ}}},\left[\mathrm{AK}, \mathrm{n}_{1}, \mathrm{t}_{\mathrm{AN}}, \mathrm{AZ}\right]_{\mathrm{K}_{\mathrm{C}}} \\
\text { The Ticket-Granting Ticket allows the client to request } \\
\text { the Service Ticket from the authorization service }\end{array}$ \\
\hline $\mathrm{R}_{3}$ & $\mathrm{C} \rightarrow \mathrm{AZ}$ & Kerberos & & $\mathrm{TGT}_{\mathrm{K}_{\mathrm{AZ}}},\left[\mathrm{C}, \mathrm{t}_{C}\right]_{\mathrm{AK}}, \mathrm{RS}, \mathrm{n}_{2}$ \\
\hline $\mathrm{R}_{4}$ & $\mathrm{AZ} \rightarrow \mathrm{C}$ & Kerberos & & $\mathrm{C}, \mathrm{ST}_{\mathrm{K}_{\mathrm{RS}}},\left[\mathrm{SK}, \mathrm{n}_{2}, \mathrm{t}_{\mathrm{AZ}}, \mathrm{RS}\right]_{\mathrm{AK}}$ \\
\hline $\mathrm{R}_{5}$ & $\mathrm{C} \rightarrow \mathrm{RS}$ & Kerberos & & $\begin{array}{c}\mathrm{ST}_{\mathrm{K}_{\mathrm{RS}}},\left[\mathrm{C}, \mathrm{t}_{C}\right]_{\mathrm{SK}} \\
\text { The Service Ticket allows to request resources from } R S\end{array}$ \\
\hline $\mathrm{R}_{6}$ & $\mathrm{RS} \rightarrow \mathrm{C}$ & Kerberos & & {$\left[\mathrm{t}_{C}\right]_{\mathrm{SK}}$} \\
\hline $\mathrm{R}_{7}$ & $\mathrm{C} \rightarrow \mathrm{RS}$ & Kerberos & & [request] $]_{\text {SK }}$ \\
\hline $\mathrm{R}_{8}$ & $\mathrm{RS} \rightarrow \mathrm{C}$ & Kerberos & & {$[\text { response }]_{\text {SK }}$} \\
\hline
\end{tabular}

Table 5: Kerberos relations.

\subsection{OSCAR}

OSCAR (Object Security Architecture for the internet of things) [48] is an architecture published in 2015. Its purpose is to provide end-to-end security, group communication, cache and asynchronous traffic in constrained environments.

To achieve its purpose, the OSCAR architecture provides an explicit authorization mechanism, a key per resource type instead of a key per resource server and a cache service. Using a key per resource type lets the client ask a key once to access the same type of resource on different entities, for example to request the temperature of every room in a house. OSCAR uses pre-shared certificates and asymmetric cryptographic keys in the resource servers to establish a DTLS connection with the authentication and authorization services, which provides encryption keys for their resources in return. Cache service and even third party servers can be used because of the object security mechanism.

\section{Entities, Principals, Domains, Relations}

Figure 7 presents the OSCAR architecture model. There are two domains:

$$
\begin{gathered}
\operatorname{Dom}(\text { client })=\{C\} \\
\operatorname{Dom}(\text { server })=\{\text { Cache, } A N, A Z, R S\}
\end{gathered}
$$

First, the resource server requests the authorization service for a key to encrypt its resources. Second, the same resource encryption key is asked by the client. Third, the client gets the resource from the resource server or a cache service.

Key management is based on public and private keys, and a shared secret given by the authorization server to the clients and resource servers. Asymmetric cryptography is used to ensure the data origin, the secret is used to derive a key to encrypt the response (along with the signature).

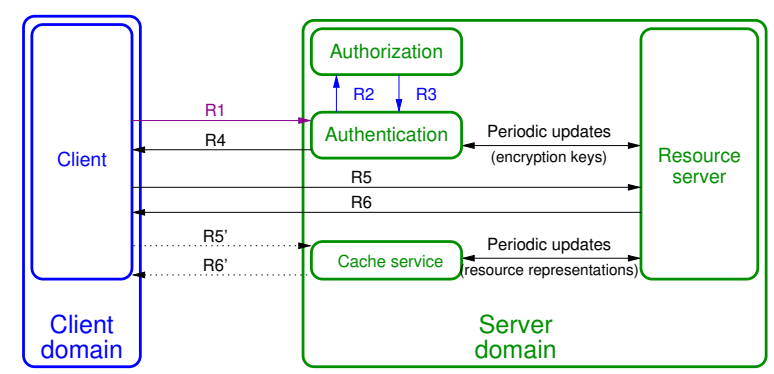

Figure 7: OSCAR architecture model.

Table 6 describes the relations in OSCAR architecture. 


\begin{tabular}{|c|c|c|c|c|}
\hline \# & entities & protocols & properties & informations \\
\hline $\mathrm{R}_{1}$ & $\mathrm{C} \leftrightarrow \mathrm{AN}$ & DTLS, CoAP & $\mathrm{F}, \mathrm{O}, \mathrm{C}, \mathrm{I}, \mathrm{MA}, \mathrm{A}, \mathrm{R}, \mathrm{AC}$ & $\begin{array}{c}\mathrm{id}_{\mathrm{C}}, \text { idproof }_{\mathrm{C}}, \mathrm{id}_{\mathrm{AN}}, \text { idproof }_{\mathrm{AN}}, \text { request } \\
\text { Identity proofs }=\text { unspecified } \\
\text { DTLS connection, abbreviated for readability reasons }\end{array}$ \\
\hline $\mathrm{R}_{2}$ & $\mathrm{AN} \rightarrow \mathrm{AZ}$ & $\perp$ & $\perp$ & $\perp$ \\
\hline $\mathrm{R}_{3}$ & $\mathrm{AZ} \rightarrow \mathrm{AN}$ & $\perp$ & $\perp$ & authorization \\
\hline $\mathrm{R}_{4}$ & $\mathrm{AN} \rightarrow \mathrm{C}$ & DTLS, CoAP & $\mathrm{C}, \mathrm{I}, \mathrm{MA}, \mathrm{R}$ & Access Secret \\
\hline $\mathrm{R}_{5}$ & $\mathrm{C} \rightarrow \mathrm{RS}$ & DTLS, CoAP & $\mathrm{C}, \mathrm{I}, \mathrm{MA}, \mathrm{R}$ & $\begin{array}{c}\text { [request }]_{\mathrm{K}_{\mathrm{i}}} \\
K=\text { key derived from Access Secret, message and certificate identities } \\
\text { No DTLS cipher negotiation: ciphers are pre-configured in certificates }\end{array}$ \\
\hline $\mathrm{R}_{5}^{\prime}$ & $\mathrm{C} \rightarrow$ Cache & DTLS, CoAP & $\mathrm{C}, \mathrm{I}, \mathrm{MA}, \mathrm{R}$ & $\begin{array}{c}\text { idproof, request } \\
\text { Idproof = key derived from Access Secret, message and certificate identities } \\
\text { No DTLS cipher negotiation: } \text { ciphers are pre-configured in certificates }\end{array}$ \\
\hline $\mathrm{R}_{6}$ & $\mathrm{RS} \rightarrow \mathrm{C}$ & DTLS, CoAP & $\mathrm{C}, \mathrm{I}, \mathrm{MA}, \mathrm{R}$ & $\begin{array}{c}{\left[[\text { response }]_{S_{R S}}\right]_{K_{\mathrm{i}}}} \\
\text { Signature performed by the private key of the resource server } \\
K=\text { key derived from Access Secret, message and certificate identities }\end{array}$ \\
\hline $\mathrm{R}_{6}^{\prime}$ & Cache $\rightarrow \mathrm{C}$ & DTLS, CoAP & $\mathrm{C}, \mathrm{I}, \mathrm{MA}, \mathrm{R}$ & $\begin{array}{c}{\left[[\text { response }]_{S_{R S}}\right]_{\mathrm{K}_{\mathrm{i}}}} \\
\text { Signature performed by the private key of the resource server } \\
K=\text { key derived from Access Secret, message and certificate identities }\end{array}$ \\
\hline
\end{tabular}

Table 6: OSCAR relations.

\section{Advantages and drawbacks}

OSCAR has several advantages: first, a simple cache system is provided. Second, the keying mechanism requires to provide only one key for a set of resources to the clients, independently of the resource servers providing them. Third, algorithm negotiation does not require additional messages, since the ciphers to use are already included in certificates.

A major drawback of OSCAR is the constant use of asymmetric cryptography to sign each resource representation, thus using time for the computation and taking 40 to 44 bytes of payload in each message. Also, the systematic use of asymmetric cryptography makes OSCAR unsuitable for low-latency applications unless resource servers are powerful enough.

\subsection{MQTT-SN}

MQTT-SN is an architecture based on MQTT, formerly called Message Queuing Telemetry Transport, but with an emphasis towards sensor networks (SN). The lastest version of MQTT-SN was published in 2013 [33] by the OASIS working group. Its purpose is to provide a straightforward publish and subscribe system, useful for data harvesting in constrained environments.

To achieve its purpose, the architecture defines a proxy, and authentication and authorization entities are separated from the resource servers. Resource servers update their resource representation to a channel handled by the proxy and followed by the interested clients.

MQTT-SN does not mention security in the reference document, however communications are likely to be secured using DTLS since this protocol is already widespread in wireless sensor networks. Several methods may be used to authenticate an entity, such as certificates, raw public key or pre-shared key, as provided by DTLS.

Entities, Principals, Domains, Relations

Figure 8 presents the MQTT architecture model. There are two domains:

$$
\begin{gathered}
\operatorname{Dom}(\text { client })=\{C\} \\
\operatorname{Dom}(\text { server })=\{\text { Proxy, } A N, A Z, R S\}
\end{gathered}
$$

The separation between the resource server and other entities implies that another device can handle heavy computations (the proxy). However, the publish subscribe mechanism implies updates, the resource server is active even if there is no client.

Table 7 describes the relations in MQTT-SN architecture.

Informations $I$ involved in these relations (apart from the DTLS informations) are: $\mathrm{I}=\{$ keep alive, protocol id, message id, flags, topic id or name, data\} 


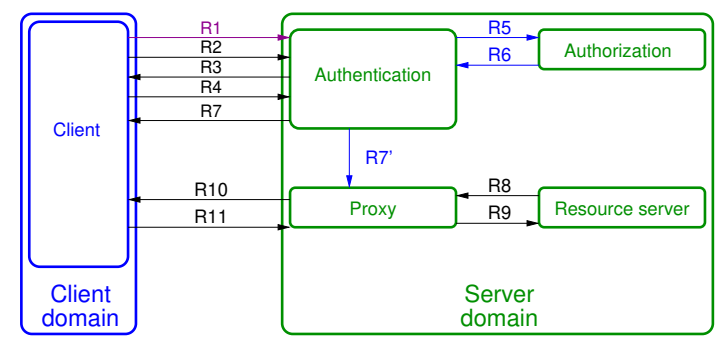

Figure 8: MQTT-SN architecture model.

- message id: identifier of the message;

- topic: interest element identifier that clients subscribe to, for example a resource name from a particular sensor such as "/sensorA/temp";

- keep alive: duration of the keep alive timer;

- data: MQTT-SN data, sensor updates.

\begin{tabular}{|c|c|c|c|c|}
\hline \# & entities & protocols & properties & informations \\
\hline $\mathrm{R}_{1}$ & $\mathrm{C} \leftrightarrow \mathrm{AN}$ & DTLS & $\mathrm{F}, \mathrm{O}, \mathrm{C}, \mathrm{I}, \mathrm{MA}, \mathrm{A}, \mathrm{R}, \mathrm{AC}$ & $\begin{array}{c}\operatorname{id}_{\mathrm{C}}, \operatorname{idproof}_{\mathrm{C}}, \mathrm{id}_{\mathrm{AN}}, \operatorname{idproof}_{\mathrm{AN}} \\
\text { DTLS connection, abbreviated for readability reasons }\end{array}$ \\
\hline $\mathrm{R}_{2}$ & $\mathrm{C} \rightarrow \mathrm{AN}$ & DTLS, MQTTSN & $\mathrm{C}, \mathrm{I}, \mathrm{MA}, \mathrm{R}$ & id $_{C}$, keep alive, protocol id, flags \\
\hline $\mathrm{R}_{3}$ & $\mathrm{AN} \rightarrow \mathrm{C}$ & DTLS, MQTTSN & $\mathrm{C}, \mathrm{I}, \mathrm{MA}, \mathrm{R}$ & return code \\
\hline $\mathrm{R}_{4}$ & $\mathrm{C} \rightarrow \mathrm{AN}$ & DTLS, MQTTSN & $\mathrm{C}, \mathrm{I}, \mathrm{MA}, \mathrm{R}$ & $\begin{array}{c}\text { msg id, topic id or name } \\
\text { The client subscribes to a topic }\end{array}$ \\
\hline $\mathrm{R}_{5}$ & $\mathrm{AN} \rightarrow \mathrm{AZ}$ & $\perp$ & $\perp$ & $\perp$ \\
\hline $\mathrm{R}_{6}$ & $\mathrm{AZ} \rightarrow \mathrm{AN}$ & $\perp$ & $\perp$ & $\begin{array}{c}\text { authorization } \\
\text { Authorization entity allows the subscription }\end{array}$ \\
\hline $\mathrm{R}_{7}$ & $\mathrm{AN} \rightarrow \mathrm{C}$ & DTLS, MQTTSN & C, I, MA, R & msg id, topic id, return code \\
\hline $\mathrm{R}_{7}^{\prime}$ & $\mathrm{AN} \rightarrow$ Proxy & DTLS, MQTTSN & $\perp$ & $\begin{array}{c}\perp \\
\text { The client is subscribed to the topic }\end{array}$ \\
\hline $\mathrm{R}_{8}$ & RS $\rightarrow$ Proxy & DTLS, MQTTSN & $\mathrm{C}, \mathrm{I}, \mathrm{MA}, \mathrm{R}$ & topic id, msg id, data \\
\hline $\mathrm{R}_{9}$ & Proxy $\rightarrow$ RS & DTLS, MQTTSN & $\mathrm{C}, \mathrm{I}, \mathrm{MA}, \mathrm{R}$ & topic id, msg id, return code \\
\hline $\mathrm{R}_{10}$ & Proxy $\rightarrow C$ & DTLS, MQTTSN & $\mathrm{C}, \mathrm{I}, \mathrm{MA}, \mathrm{R}$ & topic id, msg id, data \\
\hline $\mathrm{R}_{11}$ & $\mathrm{C} \rightarrow$ Proxy & DTLS, MQTTSN & $\mathrm{C}, \mathrm{I}, \mathrm{MA}, \mathrm{R}$ & topic id, msg id, return code \\
\hline
\end{tabular}

Table 7: MQTT-SN relations.

\section{Advantages and drawbacks}

MQTT-SN is explicitly designed for constrained environments, it has low message size overhead, a resource server only has to connect itself to a single broker and the latter will handle the requests from the clients. In this case, there is no correlation between the number of clients and the number of messages sent by the resource server.

The first drawback is the use of DTLS since it implies more than ten messages at the connection of a resource server to the proxy, then it takes up to 29 bytes of payload in each message.

Another drawback is the absence of metadata in the MQTT protocol: resources do not have types, thus a client cannot ask for a special encoding of a resource representation, such as a string representation of a temperature in a specific unit.

\subsection{OSCOAP-based architecture}

OSCOAP (Object Security of CoAP) [43] is a security mechanism based on COSE and CoAP. As said at the beginning of this section, we built minimal architectures upon security protocols by using them between only two 
devices (the client and the resource server), thus implying the resource server device to also contain authentication and authorization entities.

This simple architecture allows very basic authentication (based on the knowledge of a stored key) and authorization (with static rules for example based on the identity of the peer). Our OSCOAP-based architecture only uses CoAP and COSE (format and procedures) $\sqrt{5}$, and authorization is coarse-grained (each authenticated client can perform any request). This architecture protects the CoAP payload and all options that are not used for forwarding.

Entities, Principals, Domains, Relations

Figure 9 shows an OSCOAP architecture model. There are two domains:

$$
\operatorname{Dom}(\text { client })=\{C\}
$$

$\operatorname{Dom}($ server $)=\{$ Cache $, A N, A Z, R S\}$

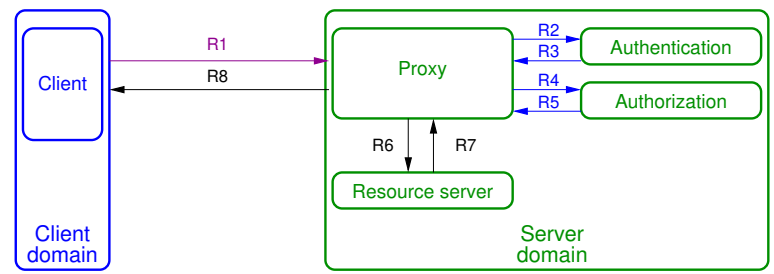

Figure 9: OSCOAP-based architecture model.

Table 8 describes the relations in OSCOAP architecture. The first message is already encrypted since the key exchange is already done.

Informations $I$ involved in these relations are: $\mathrm{I}=\left\{\mathrm{id}_{a_{b}}, \mathrm{Seq}_{a_{b}}\right.$, request, response $\}$

- $\operatorname{id}_{a_{b}}$ : identifier of $a$ as known by $b$;

- $\operatorname{Seq}_{a_{b}}$ : sequence number (from $a$ to $b$ );

- request, response: CoAP messages.

\begin{tabular}{|c|c|c|c|c|}
\hline$\#$ & entities & protocols & properties & informations \\
\hline $\mathrm{R}_{1}$ & $\mathrm{C} \rightarrow$ Proxy & CoAP & $\mathrm{C}, \mathrm{I}, \mathrm{MA}, \mathrm{R}$ & $\begin{array}{c}\operatorname{id}_{\mathrm{C}_{\text {proxy }}}, \mathrm{Seq}_{\mathrm{C}_{\mathrm{proxy}}}, \text { request } \\
\text { Request }=\text { \{URIAS, URI resource, action }\}\end{array}$ \\
\hline $\mathrm{R}_{2}$ & Proxy $\rightarrow$ AN & $\perp$ & $\perp$ & $\perp$ \\
\hline $\mathrm{R}_{3}$ & $\mathrm{AN} \rightarrow$ Proxy & $\perp$ & $\perp$ & $\perp$ \\
\hline $\mathrm{R}_{4}$ & Proxy $\rightarrow$ AZ & $\perp$ & $\perp$ & $\perp$ \\
\hline $\mathrm{R}_{5}$ & $\mathrm{AZ} \rightarrow$ Proxy & $\perp$ & $\perp$ & authorization \\
\hline $\mathrm{R}_{6}$ & Proxy $\rightarrow$ RS & $\perp$ & $\mathrm{C}, \mathrm{I}, \mathrm{MA}, \mathrm{R}$ & $\mathrm{id}_{\text {proxy }_{R S}}$, Seq $_{\text {proxy }_{R S}}$, request \\
\hline $\mathrm{R}_{7}$ & RS $\rightarrow$ Proxy & $\perp$ & C, I, MA, R & $\mathrm{id}_{\mathrm{RS}_{\text {proxy }}}, \mathrm{Seq}_{\mathrm{RS}}{ }_{\text {proxy }}$, response \\
\hline $\mathrm{R}_{8}$ & Proxy $\rightarrow C$ & $\perp$ & C, I, MA, R & $\mathrm{id}_{\text {proxy }_{C}}$, Seq $_{\text {proxy }_{C}}$, response \\
\hline
\end{tabular}

Table 8: OSCOAP relations.

\section{Advantages and drawbacks}

As the major advantage, OSCOAP architecture implies very few message exchanges because there is no mandatory handshake and key bootstrapping prior the request.

However, OSCOAP lacks denial of service protection, encrypted formed packets can be sent to overload the resource server. Also, cipher negotiation is not part of the OSCOAP document, but another draft provides a negotiation using authenticated Diffie-Hellman key exchange with ephemeral keys [42].

${ }^{5} \mathrm{COSE}$ enables CoAP end-to-end security with basic security services (confidentiality, authentication, integrity and replay protection). 


\subsection{DTLS-based architecture}

This architecture is not described in the literature: we defined it since is is likely to be deployed in practice. Our purpose is to formalize its description and include it in our comparison.

In this architecture, only two devices (the client and the resource server) exchange information using the DTLS protocol. DTLS (Datagram Transport Layer Security) [35] is an authentication protocol lastly defined in 2012. Profiles for the Internet of Things are provided by the IETF DICE working group [47]. With this architecture, authentication and authorization entities are embedded in the resource server, providing very basic services such as authentication based on the knowledge of a stored key or authorization using static rules such as for example a coarse-grained authorization (any authenticated client can perform any request) or roles based on the peer identity.

Entities, Principals, Domains, Relations

Figure 10 shows a minimal security architecture based on DTLS. There are two domains:

$$
\operatorname{Dom}(\text { client })=\{C\}
$$

$\operatorname{Dom}($ server $)=\{A N, A Z, R S\}$

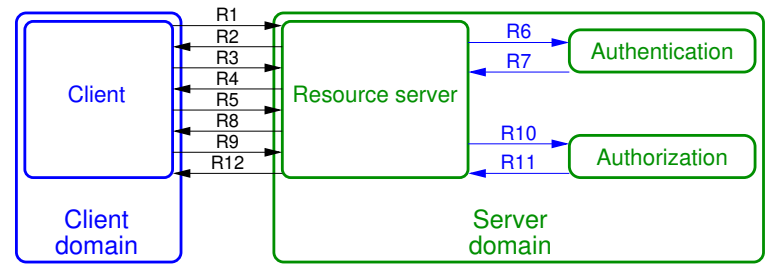

Figure 10: DTLS-based minimal architecture model.

Table 9 describes the relations in this DTLS minimal architecture.

\begin{tabular}{|c|c|c|c|c|}
\hline \# & entities & protocols & properties & informations \\
\hline $\mathrm{R}_{1}$ & $\mathrm{C} \rightarrow \mathrm{RS}$ & DTLS & & idd $_{C}$, version, id session, random 1, algo list \\
\hline $\mathrm{R}_{2}$ & $\mathrm{RS} \rightarrow \mathrm{C}$ & DTLS & & $\begin{array}{c}\text { cookie } \\
\text { RS sends a cookie, stays stateless (DoS protection) }\end{array}$ \\
\hline $\mathrm{R}_{3}$ & $\mathrm{C} \rightarrow \mathrm{RS}$ & DTLS & $\mathrm{I}, \mathrm{MA}, \mathrm{R}, \mathrm{AC}$ & id $_{C}$, version, id session, random1, algo list, cookie \\
\hline $\mathrm{R}_{4}$ & $\mathrm{RS} \rightarrow \mathrm{C}$ & DTLS & I, MA, R, AC & $\begin{array}{l}\text { version, algo, random2, (certificate, } \mathrm{DH} \text { params, accepted certificates types and principals, ) } \\
\mathrm{id}_{\mathrm{RS}}, \text { idproof }_{\mathrm{RS}}\end{array}$ \\
\hline $\mathrm{R}_{5}$ & $\mathrm{C} \rightarrow \mathrm{RS}$ & DTLS & $\mathrm{I}, \mathrm{MA}, \mathrm{R}, \mathrm{AC}$ & $\begin{array}{c}\text { id }_{C} \text {, idproof } \\
\text {, } \text { (certificate, signature, DH params, ) [data] encrypted as negotiated } \\
\text { This message ensures the negotiation went right } \\
\text { (verifies the integrity and authenticity of past messages) }\end{array}$ \\
\hline $\mathrm{R}_{6}$ & $\mathrm{RS} \rightarrow \mathrm{AN}$ & $\perp$ & $\perp$ & $\perp$ \\
\hline $\mathrm{R}_{7}$ & $\mathrm{AN} \rightarrow \mathrm{RS}$ & $\perp$ & $\perp$ & $\perp$ \\
\hline $\mathrm{R}_{8}$ & $\mathrm{RS} \rightarrow \mathrm{C}$ & DTLS & $\mathrm{C}, \mathrm{I}, \mathrm{MA}, \mathrm{R}, \mathrm{AC}$ & $\begin{array}{l}\text { [data] encrypted as negotiated } \\
\text { This message ensures the negotiation went right } \\
\text { (verifies the integrity and authenticity of past messages) }\end{array}$ \\
\hline $\mathrm{R}_{9}$ & $\mathrm{C} \rightarrow \mathrm{RS}$ & DTLS, CoAP & $\mathrm{C}, \mathrm{I}, \mathrm{MA}, \mathrm{R}$ & request \\
\hline $\mathrm{R}_{10}$ & $\mathrm{RS} \rightarrow \mathrm{AZ}$ & $\perp$ & $\perp$ & $\perp$ \\
\hline $\mathrm{R}_{11}$ & $\mathrm{AZ} \rightarrow \mathrm{RS}$ & $\perp$ & $\perp$ & authorization \\
\hline $\mathrm{R}_{12}$ & $\mathrm{RS} \rightarrow \mathrm{C}$ & DTLS, CoAP & C, I, MA, R & response \\
\hline
\end{tabular}

Table 9: DTLS relations. Optionnal informations in parenthesis.

\section{Advantages and drawbacks}

DTLS architecture has few advantages, the algorithm negotiation is simple enough for constrained environments, with only two bytes to represent a cipher suite (encryption, authentication and integrity checking algorithm, key sizes and key agreement protocol).

However, the connection implies at least 10 messages in an IEEE 802.15.4 network then 29 bytes of message size footprint. 


\subsection{IPsec-based architecture}

As with the previous architecture, we defined this one since the IPsec [23] set of protocols has been described [12] as a viable alternative solution for the Internet of Things.

Our architecture is also defined by two devices (the client and the resource server) exchanging information using the IP Encapsulating Security Payload (ESP) protocol [22, 29] to protect the payload of the network layer ${ }^{6}$ This protocol brings to our architecture confidentiality, data origin authentication, integrity verification, anti-replay protection and a limited traffic flow confidentiality. Our architecture also uses the Internet Key Exchange (v2) [20, 38] for key management and cryptographic agility $]^{7}$ As with the DTLS architecture, authentication and authorization entities are embedded in the resource server, providing very basic services.

Entities, Principals, Domains, Relations

Figure 11 shows our security architecture based on IPsec. There are two domains:

$$
\operatorname{Dom}(\text { client })=\{C\}
$$

$\operatorname{Dom}($ server $)=\{A N, A Z, R S\}$

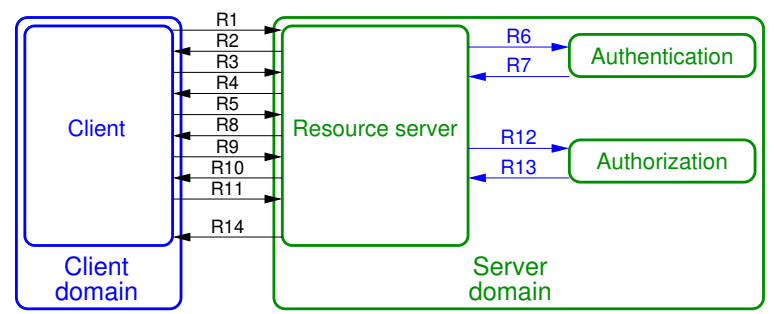

Figure 11: IPsec-based minimal architecture model.

Table 10 describes the relations.

\section{Advantages and drawbacks}

IPsec-based architecture enables fine tuning of the communication protection between two endpoints. IPsec was designed with internet communications in mind and goes as far as independently protect every communication with different secrets and algorithms, discriminating them on the IP addresses, ports or next layer protocol.

IPsec protocol is part of IPv6 standard, and all IPv6 enabled devices should implement it following the IETF recommandations [19], so IPsec-based architectures only require standard IPv6 implementations to work. However, the IETF standardization organization acknowledges that other approaches can be justified in constrained environments.

The main drawback of this architecture is its complexity due to the fine tuning, these functionalities are not relevant in constrained networks where even the size of the program binary matters and should be stripped to the bare minimum. Another drawback is the mandatory network layer. Finally, the IPsec architecture does not allow several clients on a same device: the IPsec protocol discriminates communications channels on the host identity, IP addresses, ports and next layer protocols, not on client identity.

\subsection{SSH-based architecture}

SSH (Secure SHell) [52] is a set of protocols 8 These protocols are simple and widely deployed.

\footnotetext{
${ }^{6}$ Authentication Header (AH) protocol [21,29] can also perform authentication and identity verification of the IP header, but a few drawbacks (such as NAT traversal) and the lack of use cases compared to ESP limit its use in practice.

${ }^{7}$ The IPsec protocol is not bound to IKE, other protocols can be used instead.

${ }^{8}$ First, SSH specifies a transport layer [53] to provide end-to-end encryption of the communication, algorithm negotiation, integrity, confidentiality, key exchange and server authentication. Second, SSH specifies an authentication protocol [50]. Finally SSH specifies a connection protocol [51] to provide multiplexed transport channels, and functionalities with specific purposes such as remote execution of commands.
} 


\begin{tabular}{|c|c|c|c|c|}
\hline$\#$ & entities & protocols & properties & informations \\
\hline $\mathrm{R}_{1}$ & $\mathrm{C} \rightarrow \mathrm{RS}$ & IKEv2 & & $\begin{array}{c}\mathrm{SA}_{\mathrm{C}}, \mathrm{KE}_{\mathrm{C}}, \mathrm{N}_{\mathrm{C}} \\
S A=\text { Security Association } \\
K E=\text { Key Exchange } \\
N_{C}=\text { nonce from the client } \\
\text { First message, the client connects to } R S\end{array}$ \\
\hline $\mathrm{R}_{2}$ & $\mathrm{RS} \rightarrow \mathrm{C}$ & IKEv2 & & $\begin{array}{c}\text { cookie } \\
R S \text { sends a cookie, stays stateless (DoS protection) }\end{array}$ \\
\hline $\mathrm{R}_{3}$ & $\mathrm{C} \rightarrow \mathrm{RS}$ & IKEv2 & & $\begin{array}{c}\text { cookie, } \mathrm{SA}_{\mathrm{C}}, \mathrm{KE}_{\mathrm{C}}, \mathrm{N}_{\mathrm{C}} \\
\text { C sends the cookie back with the original message }\end{array}$ \\
\hline $\mathrm{R}_{4}$ & $\mathrm{RS} \rightarrow \mathrm{C}$ & IKEv2 & & $\begin{array}{c}\mathrm{SA}, \mathrm{KE}, \mathrm{N}_{\mathrm{RS}} \\
N_{R S}=\text { nonce from the resource server } \\
R S \text { sends algorithms to use }\end{array}$ \\
\hline $\mathrm{R}_{5}$ & $\mathrm{C} \rightarrow \mathrm{RS}$ & IKEv2 & & $\begin{array}{c}\mathrm{id}_{\mathrm{C}}, \mathrm{AUTH}, \mathrm{SA}, \mathrm{TS}_{\mathrm{C}}, \mathrm{TS}_{\mathrm{RS}} \\
\text { TS = Traffic Selector } \text { (to select the traffic to encrypt } \\
\text { C authenticates itself to } R S\end{array}$ \\
\hline $\mathrm{R}_{6}$ & $\mathrm{RS} \rightarrow \mathrm{AN}$ & $\perp$ & $\perp$ & $\perp$ \\
\hline $\mathrm{R}_{7}$ & $\mathrm{AN} \rightarrow \mathrm{RS}$ & $\perp$ & $\perp$ & $\perp$ \\
\hline $\mathrm{R}_{8}$ & $\mathrm{RS} \rightarrow \mathrm{C}$ & IKEv2 & & $\begin{array}{l}\mathrm{id}_{\mathrm{RS}}, \mathrm{AUTH}, \mathrm{SA}, \mathrm{TS}_{\mathrm{C}}, \mathrm{TS}_{\mathrm{RS}} \\
R S \text { authenticates itself to } C\end{array}$ \\
\hline $\mathrm{R}_{9}$ & $\mathrm{C} \rightarrow \mathrm{RS}$ & IKEv2 & $\mathrm{C}, \mathrm{I}, \mathrm{MA}, \mathrm{R}$ & $\begin{array}{c}\mathrm{SA}, \mathrm{SA}, \mathrm{N}_{\mathrm{C}},\left(\mathrm{KE}_{\mathrm{C}},\right) \mathrm{TS}_{\mathrm{C}}, \mathrm{TS}_{\mathrm{RS}} \\
\text { First encrypted message, to create a new security association }\end{array}$ \\
\hline $\mathrm{R}_{10}$ & $\mathrm{RS} \rightarrow \mathrm{C}$ & IKEv2 & $\mathrm{C}, \mathrm{I}, \mathrm{MA}, \mathrm{R}$ & $\begin{array}{c}\mathrm{SA}, \mathrm{N}_{\mathrm{RS}},\left[\mathrm{KE}_{\mathrm{RS}},\right] \mathrm{TS}_{\mathrm{C}}, \mathrm{TS}_{\mathrm{RS}} \\
\text { Response from } R S \text {, creation of a new security association }\end{array}$ \\
\hline $\mathrm{R}_{11}$ & $\mathrm{C} \rightarrow \mathrm{RS}$ & IKEv2, CoAP & $\mathrm{C}, \mathrm{I}, \mathrm{MA}, \mathrm{R}$ & request \\
\hline $\mathrm{R}_{12}$ & $\mathrm{RS} \rightarrow \mathrm{AZ}$ & $\perp$ & $\perp$ & $\perp$ \\
\hline $\mathrm{R}_{13}$ & $\mathrm{AZ} \rightarrow \mathrm{RS}$ & $\perp$ & $\perp$ & authorization \\
\hline $\mathrm{R}_{14}$ & $\mathrm{RS} \rightarrow \mathrm{C}$ & IKEv2, CoAP & $\mathrm{C}, \mathrm{I}, \mathrm{MA}, \mathrm{R}$ & response \\
\hline
\end{tabular}

Table 10: IPsec (IKEv2) relations. Optionnal informations in parenthesis.

Our SSH architecture is based on these protocols. As said at the beginning of this section, we built minimal architectures upon security protocols by using them between only two devices (the client and the resource server), thus implying the resource server device to also contain authentication and authorization entities. This simple architecture allows very basic authentication and authorization (with static rules based on the identity of the peer for example). Besides public key and password authentication methods, this architecture also allows host-based authentication.

Entities, Principals, Domains, Relations

Figure 12 shows a minimal security architecture based on SSH. There are two domains:

$$
\operatorname{Dom}(\text { client })=\{C\}
$$

$$
\operatorname{Dom}(\text { server })=\{A N, A Z, R S\}
$$

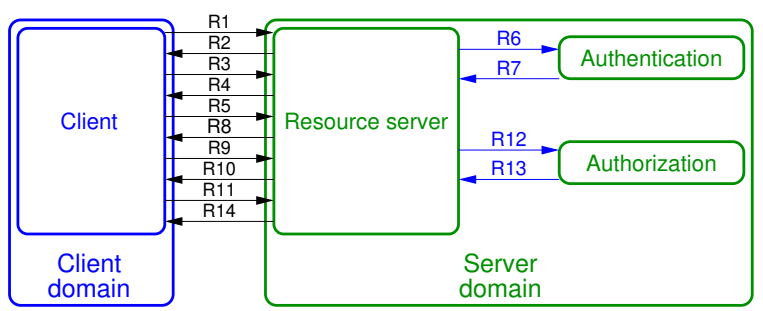

Figure 12: SSH-based minimal architecture model.

Table 11 describes the relations in SSH minimal architecture. SSH uses TCP to handle reliability and ordering. Authentication, authorization services and resource server are linked. Once the negotiation is done, a channel is created to let pass the data, this mechanism allows different data flows in a single connection. 


\begin{tabular}{|c|c|c|c|c|}
\hline \# & entities & protocols & properties & informations \\
\hline$R_{1}$ & $\mathrm{C} \rightarrow \mathrm{RS}$ & TCP, SSH & $\mathrm{F}, \mathrm{O}$ & version \\
\hline$R_{2}$ & $\mathrm{RS} \rightarrow \mathrm{C}$ & TCP, SSH & $\mathrm{F}, \mathrm{O}$ & version \\
\hline$R_{3}$ & $\mathrm{C} \rightarrow \mathrm{RS}$ & TCP, SSH & $\mathrm{F}, \mathrm{O}$ & id $_{C}$, algorithms list $C$, language ${ }_{C}$ \\
\hline$R_{4}$ & $\mathrm{RS} \rightarrow \mathrm{C}$ & TCP, SSH & $\mathrm{F}, \mathrm{O}$ & id $_{\mathrm{RS}}$, algorithms list $\mathrm{RS}$, language $\mathrm{RS}_{\mathrm{R}}$ \\
\hline$R_{5}$ & $\mathrm{C} \rightarrow \mathrm{RS}$ & TCP, SSH & $\mathrm{F}, \mathrm{O} \mathrm{AC}$ & DH params, signature \\
\hline$R_{6}$ & $\mathrm{RS} \rightarrow \mathrm{AN}$ & $\perp$ & $\perp$ & $\perp$ \\
\hline$R_{7}$ & $\mathrm{AN} \rightarrow \mathrm{RS}$ & $\perp$ & $\perp$ & $\perp$ \\
\hline$R_{8}$ & $\mathrm{RS} \rightarrow \mathrm{C}$ & TCP, SSH & $\mathrm{F}, \mathrm{O}, \mathrm{AC}$ & DH params, signature \\
\hline$R_{9}$ & $\mathrm{C} \rightarrow \mathrm{RS}$ & TCP, SSH & F, O, C, I, MA, R & channel opening \\
\hline$R_{10}$ & $\mathrm{RS} \rightarrow \mathrm{C}$ & TCP, SSH & $\mathrm{F}, \mathrm{O}, \mathrm{C}, \mathrm{I}, \mathrm{MA}, \mathrm{R}$ & channel opening confirmation \\
\hline$R_{11}$ & $\mathrm{C} \rightarrow \mathrm{RS}$ & TCP, SSH, CoAP & $\mathrm{F}, \mathrm{O}, \mathrm{C}, \mathrm{I}, \mathrm{MA}, \mathrm{R}$ & request \\
\hline$R_{12}$ & $\mathrm{RS} \rightarrow \mathrm{AZ}$ & $\perp$ & $\perp$ & $\perp$ \\
\hline$R_{13}$ & $\mathrm{AZ} \rightarrow \mathrm{RS}$ & $\perp$ & $\perp$ & authorization \\
\hline$R_{14}$ & $\mathrm{RS} \rightarrow \mathrm{C}$ & TCP, SSH, CoAP & $\mathrm{F}, \mathrm{O}, \mathrm{C}, \mathrm{I}, \mathrm{MA}, \mathrm{R}$ & response \\
\hline
\end{tabular}

Table 11: SSH relations

\section{Advantages and drawbacks}

A major drawback for SSH is the algorithm negotiation in plain text, which is not compatible with constrained networks. As an example, aes128-gcm@openssh.com is an actual encryption algorithm during a negotiation, and there are 6 types of algorithms to exchange in a single message. The use of TCP allows to fragment the message, but it is also another major drawback since TCP uses 20 bytes of each packet payload.

\section{Comparison}

To compare all these architectures, we now detail the two scenarios briefly mentioned in the introduction.

\subsection{Scenarios}

Depending on the architecture and its deployment, key management and authentication procedure may vary. For example, the client can or cannot be reachable from the server domain principal: this principal can or cannot change the cryptographic material on the client at any time. Thus, the principal may want to limit the validity period of the cryptographic material of the client.

The following scenarios cover frequently used authentication (pre-shared keys, certificates) mechanisms, with a client outside and inside the server domain.

In the first scenario, an external client entity without constraints requests a resource server within our server domain LAN. The architecture needs mutually authenticated client and authentication entity, an authorization entity granting permissions (and allowed operations) for a request, and secure communications (confidentiality, authenticity and integrity). In most cases, authentication (and authorization) is performed through asymmetric cryptography (a couple of public and private keys on each side), with the elliptic curve digital signature authentication algorithm (ECDSA) and communication is secured through symmetric cryptography (detailed in section 4.2). In the first scenario, the key to protect the communication is provided by the ephemeral elliptic curve Diffie-Hellman (ECDHE) ${ }^{9}$ when possible. Certificates and Diffie-Hellman algorithm allow the resource server not to retain information about each client so it is useful when there is a large number of clients.

In the second scenario, the client entity is on a constrained device in the server domain LAN, and pre-shared keys will be preferred for the authentication. Every resource server is contacted by a limited number of clients, thus allowing the resource servers to have a pre-shared key for each of them, and vice versa.

Some architectures do not have mechanisms for the first scenario (e.g.: Kerberos, since it was not initially designed to handle public key cryptography) or are not well suited for the IEEE 802.15.4 MTU (e.g.: OSCOAP, in the first scenario with its COSE-based message format).

\footnotetext{
${ }^{9}$ Currently recommended elliptic curves provided by [25].
} 


\subsection{Communication stack}

Our definition of security architecture is unrelated to a particular link, network or transport layer. For the comparison, these parameters are fixed and based on constrained environment standards, unless otherwise specified in the architecture.

The physical and link layers used in this comparison are IEEE 802.15.4 [14]; this is the standard in constrained environments in the literature and the hardware is easily available. This technology only allows 127 bytes of payload, minus a minimal 9-bytes link layer header.

The network layer used for this comparison is IPv6 over Low power Wireless Personal Area Networks (6LoWPAN) [30], which is the introduction of IPv6 in constrained networks. For communication between two devices in the same network ${ }^{10}$, it only adds a 2-byte header for the network layer if there is only one hop to the 6LoWPAN router, and the header takes 7 bytes if there are multiple hops. For the sake of our comparison, entities are considered at a single hop from the border router. This leaves 116 bytes for next parts.

The transport layer used is UDP when possible with the truncated version provided by 6LoWPAN taking 4 more bytes. When TCP is required by the architecture, 20 bytes are used. This leaves 112 bytes for next parts (96 with TCP).

Table 12 summaries the remaining packet size for the application protocol in an IEEE 802.15.4 network using a 9-bytes link layer header and 6LoWPAN, depending on the context of the communication (endpoints in the same network, in same network but with multi-hops from the border router or in different networks).

\begin{tabular}{|c|c|c|c|}
\cline { 2 - 4 } \multicolumn{1}{c|}{} & same network & $\begin{array}{c}\text { same network } \\
\text { multi hops }\end{array}$ & different networks \\
\hline none & 116 & 109 & 98 \\
\hline UDP & 112 & 105 & 94 \\
\hline TCP & 96 & 89 & 78 \\
\hline
\end{tabular}

Table 12: Summary of remaining packet size for application layer depending on the used transport protocol and the network topology in a 6LoWPAN network with IEEE 802.15.4 link layer.

The application layer used for this comparison is CoAP. This protocol is designed by the IETF CoRE working group specifically for constrained environments: simple, low header overhead and parsing complexity. CoAP provides (i) proxy (and cache) support; (ii) observation support, which means a client can receive notifications when a resource changes; (iii) resource discovery; and (iv) group communication (through multicast support). Resource discovery is not used in this comparison since it is not in the scope of this documen 11 CoAP has a 4-byte header, and two more bytes will be used for the Content-Format option.

About the security in this comparison, we have chosen AES 128 bits in Counter CBC-MAC (CCM) mode which is an Authenticated Encryption with Additional Data (AEAD) algorithm with 64 bits of integrity checking value (ICV). Kerberos and SSH-based architectures do not have this algorithm. SSH may be used with another AEAD algorithm such as AES 128 GCM [15], but there is no AEAD algorithm for Kerberos, which can instead be used with AES 128 bits in ciphertext stealing (CTS) mode along with keyed-hash message authentication code (HMAC) SHA256 for integrity verification, requiring 16 bytes of integrity checking value.

Figure 13 shows the communication stack used in our comparison without security. In a few architectures, some of these layers may change: for example MQTT-SN uses its own application layer.

\subsection{Results}

The comparison is based on security, constraints and few general aspects of the architectures, as described below and discussed in the next section. Tables 13 and 14 show a summary.

Security aspect. The first criterion is the protection of communications.

\footnotetext{
${ }^{10} 6$ LoWPAN also provides header compression for communication between devices in different networks. The header is then only reduced to 20 bytes in the worst case scenario (the prefix of the external device is unknown)

${ }^{11} \mathrm{~A}$ secure resource discovery with CoAP is still to be defined. The security of CoAP is based on DTLS, but there is no DTLS-secured group communication solution at this time [34] which could be required for resource discovery.
} 


\begin{tabular}{|c|}
\hline CoAP \\
\hline UDP \\
\hline IPv6 (6LoWPAN) \\
\hline IEEE 802.15.4 MAC \\
\hline IEEE 802.15.4 PHY \\
\hline
\end{tabular}

Figure 13: Communication stack used for comparison.

\begin{tabular}{|c|c|c|c|c|}
\hline & \\
\hline & OSCAR & ACE-DCAF & ACE-OAuth & Kerberos \\
\hline entities in client domain & client & client, AN, AZ & client & client \\
\hline entities in server domain & RS, AN, AZ, Proxy & $\mathrm{RS}, \mathrm{AN}, \mathrm{AZ}$ & $\mathrm{RS}, \mathrm{AN}, \mathrm{AZ}$ & $\mathrm{RS}, \mathrm{AN}, \mathrm{AZ}$ \\
\hline \multicolumn{5}{|l|}{ Security of the communication } \\
\hline replay protection & yes & yes & yes (via DTLS and/or COSE) & yes \\
\hline denial of service protection & none & cookie (DTLS) & none (unless DTLS is used) & none \\
\hline metadata encryption & no (payload CoAP) & yes & no (data) (unless DTLS is used) & no (data) \\
\hline cryptographic agility & $\begin{array}{l}\text { no } \\
\text { algorithms embedded in } \\
\text { preshared certificates }\end{array}$ & yes & no (unless DTLS is used) & yes \\
\hline \multicolumn{5}{|l|}{ Security elements } \\
\hline asymmetric cryptography (RS) & yes & no & no (unless DTLS is used) & no \\
\hline explicit authorization & yes & yes & yes & yes \\
\hline authorization granularity & resource type & resource & resource & resource server \\
\hline automatic key management & yes & yes & yes & yes \\
\hline context per client (RS) & no & yes & yes (via DTLS and/or COSE) & yes \\
\hline \multicolumn{5}{|l|}{ Constraints aspects } \\
\hline relation count between domains & $4+$ DTLS & 4 + DTLS + (D)TLS (+TCP) & $4+$ DTLS & 6 \\
\hline real-time clock required (RS) & yes & no & no & yes \\
\hline data cache service & yes & no & no & no \\
\hline message size overhead & $\begin{array}{c}\text { high } \\
\text { signature } \\
(40-44 \text { bytes })\end{array}$ & $\begin{array}{l}\text { medium } \\
\text { DTLS } \\
\text { (29 bytes) }\end{array}$ & $\begin{array}{c}\text { high } \\
\text { COSE }(+ \text { DTLS }) \\
(>20+29 \text { bytes })\end{array}$ & $\begin{array}{c}\text { very high } \\
>127 \text { bytes at con. }\end{array}$ \\
\hline msg count at first connection (RS) & 0 (Proxy) & $\begin{array}{c}10 \\
\text { (minimal msg count for DTLS) }\end{array}$ & $\begin{array}{c}\text { 2-4 (COSE, no EDHOC) } \\
12-16 \text { (DTLS + introspect) }\end{array}$ & $\begin{array}{l}4 \\
4\end{array}$ \\
\hline $\begin{array}{l}\text { msg count at first connection } \\
\text { (Client) }\end{array}$ & 2 & $\begin{array}{c}12 \\
\text { (DTLS + authorization request) }\end{array}$ & 4 (without DTLS) & 4 \\
\hline msg count at each request (RS) & 2 & 2 & 2-4 (without DTLS) & 2 \\
\hline msg count at each request (Client) & 2 & 2 & 2-4 (without DTLS) & 2 \\
\hline maintained connections (RS) & 1-2 (AuthN and Proxy) & 1 (AuthN+Z) & $0-1$ (introspection) & 0 \\
\hline msg count overhead (RS) & $\begin{array}{c}\text { depend on } \\
\text { update frequency }\end{array}$ & $\begin{array}{l}\text { depend on } \mathrm{nb} \\
\text { of clients and } \\
\text { request freq. }\end{array}$ & $\begin{array}{l}\text { depend on } \mathrm{nb} \\
\text { of clients and } \\
\text { request freq. }\end{array}$ & $\begin{array}{l}\text { depend on } \mathrm{nb} \\
\text { of clients and } \\
\text { request freq. }\end{array}$ \\
\hline \multicolumn{5}{|l|}{ General aspects } \\
\hline protocols used L & DTLS + CoAP & (D)TLS $(+$ TCP $)+$ CoAP & (OS)CoAP (+ DTLS) & Kerberos (+ (D)TLS) \\
\hline specific application architecture & yes & yes & yes & no \\
\hline resource discovery & could be impl. on Auth & no & no & no \\
\hline
\end{tabular}

Table 13: Comparison on security and constraint aspects of the architectures.1.1

means an advantage based on the selected metric, red means the opposite (or the result depends on the deployment). 


\begin{tabular}{|c|c|c|c|c|c|}
\hline & MQTT-SN & DTLS & IPsec & SSH & OSCOAP \\
\hline entities in client domain & client & client & client & client & client \\
\hline entities in server domain & RS, AN, AZ, Proxy & $\mathrm{RS}, \mathrm{AN}, \mathrm{AZ}$ & $\mathrm{RS}, \mathrm{AN}, \mathrm{AZ}$ & $\mathrm{RS}, \mathrm{AN}, \mathrm{AZ}$ & RS, AN, AZ, Proxy \\
\hline \multicolumn{6}{|l|}{ Security of the communication } \\
\hline replay protection & yes & yes & yes & yes & yes \\
\hline denial of service protection & cookie & cookie & cookie & yes (TCP) & none \\
\hline metadata encryption & yes & yes & yes & yes & no (only data) \\
\hline cryptographic agility & yes & yes & yes & yes & no \\
\hline \multicolumn{6}{|l|}{ Security elements } \\
\hline asymmetric cryptography & no & yes * & yes * & yes & yes * \\
\hline explicit authorization & yes & no & no & no & no \\
\hline authorization granularity & resource & resource server & resource server & resource server & resource server \\
\hline automatic key management & yes & yes & yes & yes & yes \\
\hline context per client on RS & no & yes & yes & yes & yes \\
\hline \multicolumn{6}{|l|}{ Constraints aspects } \\
\hline relation count between domains & $6+$ DTLS & 8 & 10 & $10+\mathrm{TCP}$ & 2 \\
\hline real-time clock required (RS) & no & yes * & yes * & yes * & yes * \\
\hline data cache service & yes & no & no & no & no \\
\hline message size overhead & $\begin{array}{c}\text { high } \\
\text { DTLS header } \\
\text { (29 bytes) }\end{array}$ & $\begin{array}{c}\text { high } \\
\text { DTLS header } \\
\text { (29 bytes) }\end{array}$ & $\begin{array}{c}\text { medium } \\
\text { ESP } \\
\text { (18 bytes w/o padding) }\end{array}$ & $\begin{array}{c}\text { high } \\
\text { TCP, SSH header } \\
(20+20 \text { bytes })\end{array}$ & $\begin{array}{c}\text { very high } \\
>127 \text { in scenario } 1\end{array}$ \\
\hline msg count at first connection (RS) & 14 & $14 * *(10)$ & 8 & $11(\mathrm{TCP}+\mathrm{SSH})$ & 2 \\
\hline msg count at first connection (Client) & $18 * *(14)$ & $14 * *(10)$ & 8 & $11(\mathrm{TCP}+\mathrm{SSH})$ & 2 \\
\hline msg count at each request (RS) & $\begin{array}{c}0 \text { (asynchronous) } \\
1 \text { to } 4 \text { packets / update }\end{array}$ & 2 & 2 & 2 & 2 \\
\hline msg count at each request (Client) & $\begin{array}{c}0 \text { (asynchronous) } \\
1 \text { to } 2 \text { packets / update }\end{array}$ & 2 & 2 & 2 & 2 \\
\hline maintained connections (RS) & 1 (AuthN+Z+Proxy) & 0 & 0 & 0 & 0 \\
\hline msg count overhead (RS) & $\begin{array}{c}\text { depend on } \\
\text { update frequency }\end{array}$ & $\begin{array}{l}\text { depend on } \mathrm{nb} \\
\text { of clients and } \\
\text { request freq. }\end{array}$ & $\begin{array}{l}\text { depend on } \mathrm{nb} \\
\text { of clients and } \\
\text { request freq. }\end{array}$ & $\begin{array}{l}\text { depend on } \mathrm{nb} \\
\text { of clients and } \\
\text { request freq. }\end{array}$ & $\begin{array}{l}\text { depend on } \mathrm{nb} \\
\text { of clients and } \\
\text { request freq. }\end{array}$ \\
\hline \multicolumn{6}{|l|}{ General aspects } \\
\hline protocols used L & DTLS + MQTT-SN & DTLS + CoAP & IPsec + CoAP & $\mathrm{TCP}+\mathrm{SSH}+\mathrm{CoAP}$ & CoAP (+ DTLS) \\
\hline specific application architecture & yes & no & no & no & no \\
\hline resource discovery & no & no & no & no & no \\
\hline
\end{tabular}

Table 14: Comparison on security and constraint aspects of the architectures. * changes in scenario 2 . ** plus the packets to exchange the certificates. Green means an advantage based on the selected metric, red means the opposite (or the result depends on the deployment). 
Cryptographic algorithms (such as AEAD algorithms) cover the confidentiality, authenticity and integrity protection for exchanges between entities. Also, other mechanisms supplement cryptography to protect communications, such as replay and denial of service (DoS) protection. Replay protection is always provided with sequence numbers, which is simple and efficient. Denial of service protection prevents the server from being overloaded by forcing the initiator of the connection to perform an action such as reflect an information (known as a cookie) or compute something (puzzle) before the server performs heavy computation or retain information about the client. Another criterion is the metadata encryption: architectures may use protocols that encrypt the application layer (here, CoAP) to hide metadata (who is requesting what and when). IPsec encrypts everything above the network layer, SSH and every DTLS-based architecture (ACE DCAF, MQTT-SN... ) encrypt above the transport layer, and other architectures such as OSCAR only encrypt the application layer payload. Finally, another mechanism is the cryptographic agility useful for heterogeneous deployments and to migrate from one algorithm suite to another (with stronger cryptography), over time [13].

The asymmetric cryptography on $R S$ criteria shows the architectures in which the resource server must perform heavy computations, such as authentication with certificates. For example in the OSCAR architecture each resource representation is signed by the resource server, and in our DTLS-based architecture authentication is performed by the resource server which implies asymmetric cryptography in the first scenario due to the use of certificates and DiffieHellman algorithm. When an architecture mandates the use of asymmetric cryptography, red color is used, when there is no need for it, green color is used, when the choice is up to the deployment requirements there is no color.

Authorization is explicit if the architecture defines specific messages (or informations) for the authorization mechanism (with a dedicated authorization server for example). If not, the architecture only provides coarse-grained authorization or relays this mechanism to the application layer. Depending on the granularity of the authorization, the access may be granted to a resource server (coarse grained), to a resource type or directly to a resource on a resource server (fine grained).

Automatic key management may be achieved via Diffie-Hellman algorithm or another mechanism. For example, the DTLS protocol uses random numbers in combination with cryptographic material such as pre-shared keys to create new session keys at each connection, without requiring heavy computation.

Keeping a context per client (on the resource server) allows a unique security association between two entities. Such context is composed of the current sequence numbers, negotiated algorithms, current data encryption keys and other pieces of information required for a secure communication. There is no specific context between the client and the resource server in OSCAR since the encryption key of a resource type is the same for every client. The MQTT-SN architecture has a dedicated authentication server on a powerful device, leveraging the cost of authentication on the resource server that then only needs to authenticate itself to the authentication server.

End-to-end secure communication means that the data is secured from the endpoint that holds the data to the endpoint that wants it. For example, the data can be encrypted by the resource server and can only be decrypted by the client (intermediaries cannot decrypt it). End-to-end security is important to limit the attack surface: only the resource server needs to be secured in the server domain. However, end-to-end security has a cost: the resource server needs to keep a security association for each client, implying a larger memory footprint. This can be leveraged if necessary by renewing security associations at each request, trading memory for added computation and/or messages.

Constraint aspects. Relation count between domains may help deduce the number of messages between the client and the server domains. This correlates mostly to the exchanges between two devices, showing the least number of messages exchanged for a request (messages exchanged in a given domain could be processed by a single device).

The real-time clock requirement defines the type of device on which the architecture can run. Kerberos architecture uses timestamps even on the resource server, time synchronisation between entities is required.

Data cache service indicates architectures that provide a cache for data besides a potential CoAP caching proxy.

Message size during the communication is an important factor since there is a limited maximal transfer unit. Kerberos uses ASN.1 message format along with many required informations, some with arbitrary length, the result is that messages at the connection have a size well above the MTU of IEEE 802.15.4. DTLS-based architectures take 29 bytes of payload for the security layer and 8 bytes fewer when TLS is used. COSE-based architectures let applications exchange a message with self-contained security, without connection, so for exchanging a signed message secured with ECDHE the overhead entails a considerable additional cost. However, COSE-based architectures also can reuse security associations, thus without performing ECDHE exchange multiple times.

In our examples, 6LoWPAN is used as the network layer. This protocol has optimisations for the message size, 
such as 6LoWPAN-GHC [5] that uses a compression algorithm to limit the overhead, trading message size for memory and computation in some contexts. The header compression can be efficient even when the message is encrypted with DTLS since it removes a redundancy in the DTLS header. Message sizes should be qualified in this regard.

Message count is an important value for battery-powered devices. Persistent connections between entities in an architecture are not counted since they only happen once when the device starts.

The number of exchanged messages depends on:

- the number of clients and their request frequency if there is no cache service;

- update frequency if there is a cache service and the message exchanges are reduced to unconditional updates of resource representations towards the cache service.

Notes on message counts: msg count at first connection $(R S)$ only includes the messages from the connection, not the actual request and response. A DTLS connection implies at least 10 messages (without certificates) and up to 14 messages before any data being transferred. When the resource server is expected to exchange messages with an authentication or authorization service, this communication channel is considered already open (no DTLS connection), this is why ACE OAuth introspection only adds 2 messages.

General aspects. Specific application architecture criterion means that the architecture is bound to a specific application protocol. This is the case for MQTT-SN that defines its own application protocol for instance.

Finally, resource discovery mechanism provided directly by the architecture allows a client to save some time and energy finding resources. 6LoWPAN associated with the CoAP protocol allows a client to perform a resource discovery, but this requires multicast messaging which is currently performed without security, and may not be allowed in the network.

Next section shows advantages and drawbacks of the different architectures under different situations.

\section{Which architecture given my constraints?}

We describe in this section the relevant architectures according to the potential constraints upon constrained devices. For example, an architecture can be efficient for a deployment with many clients because of a non-constrained proxy (or cache service) in charge of the message distribution, but inefficient on latency because every message is signed using asymmetric algorithms. The following recommendations are based on comparison tables explained in the previous section.

\subsection{Unsuitable architectures for constrained environments}

This analysis is focused on networks with a seriously limited maximum transfer unit (such as IEEE 802.15.4 with a maximum of 127 bytes) and potentially battery-powered devices, thus the number of transmitted messages should be as minimal as possible. Also, we focus on cheap hardware for resource servers, meaning that the fewer the functionalities required and the lower memory usage, the better.

\subsubsection{Kerberos}

The main problem which prevents the use of Kerberos in constrained environments is its message sizes. The message format is ASN.1 which adds a few bytes to every encoded information: for example a single 1-byte constant takes 5 bytes once encoded, and 19 bytes for a timestamp (the format is human readable, so inefficient in memory, computationally, in the code size and towards the MTU). During the authentication phase, messages are well above the MTU, which would require the 6LoWPAN border router and resource servers to perform IP fragmentation, or using a transport layer enabling fragmentation such as TCP (and taking 20 bytes per message ${ }^{12}$. During the communication between the client and the resource server, Kerberos is using at least 72 bytes of payload, meaning that most of the payload is taken by a protocol used only to protect the data and again, fragmentation is necessary: given a 127-byte MTU, 23 bytes of L2 header, a 20-byte 6LoWPAN header, a 4-byte UDP header, a 72-byte Kerberos header and finally

\footnotetext{
${ }^{12}$ Even on less constrained networks such as Ethernet, Kerberos has a MTU problem and TCP is used to overcome this problem.
} 
a 4-byte CoAP header, the payload should only take 4 bytes. Thus, the message format is unsuitable for constrained environments with limited MTU.

The second problem with Kerberos is that it requires real-time clocks on both the client and the resource server since timestamps are used for authentication, and to verify the request validity (authorization is time limited). Realtime clocks are not always available on very constrained hardware, and resource servers have to synchronize their clock regularly to handle a time shift.

Third, the authentication entity is separated from the resource server, meaning that authentication could be handled by a non constrained hardware. However, no certificate-based authentication is provided by Kerberos, meaning that communication should be protected by a password which is cumbersome in the first scenario.

Finally, Kerberos requires memory on the resource server: to maintain a context per client, and to store many informations required for authentication such as domain names, arbitrary names, several time stamps...

In conclusion, Kerberos architecture as originally specified is not compatible with low MTU, requires clocks and time synchronization on resource servers, is not suitable for the first scenario and requires many informations. Since the only advantage it provides compared to other architectures is the separation of authentication and authorization, which is not required by any of our scenarios, this architecture is unsuitable for constrained networks.

\subsection{2. $S S H$}

The SSH set of protocols is widespread, has a simple design, and it is useful for many applications but it has major drawbacks for constrained environments.

First, the SSH protocol has a mandatory asymmetric algorithm for authentication and key exchange even for a client in the server domain network. This implies heavy computations on the resource server at the connection establishment: this takes dozens of seconds for key agreement on a $8 \mathrm{MHz} \mathrm{CPU}$ even using elliptic curves cryptography with now insufficient cryptographic key length [26 ${ }^{13}$ Mandatory public key cryptography forces constrained nodes to have special hardware, increasing the cost of the deployment.

Second, the architecture requires the TCP transport layer implying message size overhead (20-byte TCP header), and a large message count for the handshake.

Finally, the SSH architecture like other architectures based on a single protocol, lacks a central authentication management, requiring each resource server to store information about each client. Thus, the architecture only works if the deployment is composed of resource servers with few clients each and with few simultaneous established connections due to memory constraints.

In conclusion, the SSH architecture does not stand out from other architectures regardless of the metric: message sizes, message count, cryptography used, functionalities required on resource servers, or memory used.

\subsubsection{IPsec}

The IPsec protocol is designed to protect different communications (discriminated from their IP address, ports or protocol) with different secrets and algorithms. This goal is intended for Internet communications, to protect various and unsecured communications between distant nodes. In constrained environments, each resource server has a single task only, so the discrimination will only happen on the IP address, which is useless in our case: a single security association is enough between a client and a resource server that only perform a single task. Finally, the IPsec specific functionalities are not useful in constrained environments such as described in this article, its usage could still happen between firewalls but this is out of scope.

In conclusion, whatever the constraints upon the devices, neither Kerberos, SSH nor IPsec architecture is recommended. Kerberos has a very important message size problem, SSH requires asymmetric algorithms and IPsec functionalities are not relevant in constrained environments.

\footnotetext{
${ }^{13}$ The 160-bit elliptic curves cryptography key length used in [26] is equivalent to a 1024-bit RSA key [49], which is now obsolete. The NIST recommends at least a key size of 224 bits for elliptic curves algorithms [2], thus rendering key agreement impractical on constrained nodes without the help of a special hardware to handle cryptographic operations.
} 


\subsection{Constraints on the devices}

Each deployment is built with a particular objective, but all deployments share common parameters (and potential constraints): number of clients, request frequency and intended authorization grain for example. Architectures are sorted based on their relevance to these parameters.

\section{Large number of clients}

For deployments with a large number of clients, architectures with a proxy (MQTT-SN and OSCAR) have an advantage since the number of clients and the number of messages transmitted to resource servers can be decorrelated (with a cache for example). Therefore, the proxy device can also perform authentication and authorization, and thus resource servers do not have to store information about each client, alleviating their memory consumption.

\section{High request frequency}

For deployments with high request frequency (with quickly staled sensor values), having a cache is not enough: requests latency becomes an important metric. The most suitable architectures in this context are the ones harvesting the data in a cache proxy before being requested by users (OSCAR, MQTT-SN): there is no authentication between users and resource servers (fewer generated messages, less memory constraints on resource servers, fewer connections to maintain). Architectures without a cache are the less suitable. Parameters such as the use of asymmetric cryptography and the number of relations between domains are taken into account to choose the right architecture, from the most suitable to the less: ACE DCAF (no asymmetric cryptography), ACE OAuth (no asymmetric cryptography unless DTLS is used to contact RS), then OSCOAP (requires asymmetric cryptography, but only two messages are required per request) and finally DTLS and IPsec which both require asymmetric cryptography.

Deployments without a proxy device (we assume that the proxy performs caching)

For deployments with independent nodes without available proxy device (e.g.: a stand alone light bulb at home), the available memory on resource servers is the problem. In this case, resource servers have to (i) memorize identity and cryptographic material of each client, and (ii) they have to handle multiple running connections in parallel with the related informations (cryptographic material, state, timers and so on).

OSCAR provides a simple mitigation for (i) and (ii): the architecture requires resource servers to store only one key for each resource type and not for each client. Each architecture can remove the security association after each request, allowing resource servers to only store few contexts (negotiated algorithms and keys, flags, sequence numbers and so on), this is the most efficient way to mitigate (ii). Unfortunately OSCAR requires an authentication entity to handle the clients and decrease the workload on the resource server. Thus the only way for an architecture to stand out is to have fewer transmitted messages between domains (communication with the authentication entity included).

In deployments of independent resource servers (i.e. with no server performing central authentication nor central authorization services), communication can be reduced to a simple security communication protocol. Most of the architectures use the DTLS protocol to securely exchange messages between domains: OSCAR, ACE DCAF, ACE OAuth, MQTT-SN, OSCOAP (at least in the first scenario) and the eponymous architecture. Apart from the last one, these architectures only imply more messages than the DTLS protocol and thus they have limited relevance. Remaining architectures are DTLS and IPsec, OSCOAP may be used but only with pre-shared keys.

\section{Intended authorization granularity}

Granularity of the authorization varies according to the architecture. ACE DCAF, ACE OAuth and MQTT-SN have an authorization granularity down to the resource. OSCAR grants permissions to clients with a granularity down to a resource type on all resource servers at once, but this system implies an unique key shared with many devices: a single resource server with a vulnerability can affect the security of all the resource servers. Architectures based on a single protocol (DTLS, IPsec and OSCOAP) delegate the authorization to the application, the granularity can be down to the resource but configured on each resource server which can be cumbersome for large networks.

\section{Deployments with actuators}

Requesting a data from a resource server is no different from sending a command to an actuator for all architectures except MQTT-SN which does not use CoAP. MQTT-SN is poorly suitable for deployments with actuators since clients are not expected to directly send messages to resource servers. MQTT-SN is designed as a pub-sub system for data harvesting and not as a request-response system: clients ask to receive updates of a resource, they do not send a value. Thus MQTT-SN cannot be used in a deployment with actuators as-is, resource servers with actuators need to register themselves as a client of a topic to the proxy, and vice-versa for the client in order to receive messages, this limitation implies more control messages. 


\section{Deployments with heterogeneous network}

Devices communicating with each other need to use the same cryptographic algorithms: this can be achieved with a negotiation or with a prior agreement for example. Any architecture using a negotiation protocol (DTLS, IPsec) is suitable for heterogeneous networks (eponymous architectures, ACE DCAF, MQTT-SN). OSCAR and OSCOAP require a prior agreement, which may be cumbersome with large networks of devices communicating with each other. ACE OAuth also requires a prior agreement between clients and the authentication entity unless DTLS or EDHOC are used.

Besides the security perspective and constraints on devices, MQTT-SN is unsuitable for deployments with heterogeneous softwares working with different representations of the same resource type since there is no negotiation of the resource type representation.

\subsection{Constraints on the nodes}

In constrained environments, devices have low capabilities. In the following, we present some recommendations according to the device limitations.

\section{Real-time clock}

In the first scenario, when clients are out of the server domain network, certificates may be used in order to limit the period of validity of a key. This implies the use of real-time clocks on resource servers unless the authentication entity is on another device (a proxy). OSCAR is the only architecture requiring the use of certificates and thus realtime clocks on every device. Any other architecture is suitable for deployments without real-time clock equipped devices for the second scenario, and the first if authentication with pre-shared keys is possible with outsider clients.

\section{Memory}

Deployed devices may have less than 10 kilobytes of RAM and less than 100 kilobytes of Flash memory. A few parameters consume memory such as protocols and algorithms used, client (or proxy) authentication credentials and session information.

Table 15 presents insights about memory consumption on the architectures. Only OSCAR and MQTT-SN do not require the resource server to store information about each client. Every architecture is using CoAP as the application protocol except MQTT-SN which uses a simpler protocol. Architectures are using DTLS as a negotiation protocol and secure layer except IPsec. OSCOAP and ACE OAuth architectures can avoid implementing DTLS if a prior agreement was made. ACE OAuth and OSCOAP require COSE procedures, ACE OAuth implements OSCOAP, it is by definition more memory consuming. OSCAR is the only architecture requiring asymmetric cryptographic algorithms. Finally, ACE DCAF, ACE OAuth and OSCAR require authorization procedures.

MQTT-SN is the less memory consuming, it only requires DTLS and MQTT-SN protocol, without storing a context per client. ACE DCAF and ACE OAuth have an authentication server (that can be centralized on an unique device for the whole network) to store information about clients. These architectures only requires the resource server to store session informations for established connections, they are the second less memory consuming architectures. In deployments with a great number of clients, OSCAR stands out in the remaining architectures since there is a cache service. Finally, other architectures have a similar memory consumption ${ }^{14}$

\begin{tabular}{|l|c|c|c|c|c|}
\cline { 2 - 5 } \multicolumn{1}{c|}{} & Context per client & Application protocol & Security protocol or procedure & Asymmetric cryptography & Authorization \\
\hline OSCAR & no & CoAP & DTLS & yes (and certificates) & yes \\
\hline ACE DCAF & yes (once connected) & CoAP & DTLS & yo \\
\hline ACE OAuth & yes (DTLS or COSE) & CoAP & optionally DTLS, OSCOAP & optionally \\
\hline MQTT-SN & no & MQTT-SN & DTLS & optionally & yes \\
\hline DTLS & yes (without cache) & CoAP & DTLS & optionally \\
\hline IPsec & yes (without cache) & CoAP & IPsec & no \\
\hline OSCOAP & yes (without cache) & CoAP & optionally DTLS, COSE & optionally \\
\hline
\end{tabular}

Table 15: Memory consumption: algorithms and protocols required by architectures.

\footnotetext{
${ }^{14} \mathrm{CoAP}$ allows architectures to have cache services, in that case the connection endpoint may be terminated before resource servers, alleviating the communication burden and lowering the memory footprint.
} 


\section{Computation and batteries}

Heavy computation, such as with asymmetric algorithms, induces battery depletion and thus should be avoided. However a trade-off between computation and memory can be achieved: when asymmetric cryptography is solely used for the creation of the communication channel (key agreement or authentication), the channel can be kept open.

MQTT-SN does not require asymmetric algorithms for the resource servers. However, MQTT-SN is inefficient when there is no client for a resource because resource servers perform resource updates anyway. Update frequency depends on the resource changes and the configuration.

DTLS, IPsec and OSCOAP also require asymmetric algorithms but only for authentication and in the first scenario (clients out of the server domain network).

OSCAR requires the use of asymmetric algorithms: when a resource is changed, this implies that the resource server processes a signature which is time and energy consuming, no possible trade-off.

\subsection{Summary}

Table 16 presents a summary of the previous comparisons. None of the architectures stands out in every situation. However, architectures with a proxy which authenticates users and caches responses from resource servers tend to be suitable. Depending on the architecture, the proxy hides the complexity of user management from the resource servers point of view and vice versa, data is cached and security algorithms are different on users and resource servers.

\begin{tabular}{|c|c|c|c|}
\hline & most suitable & suitable & poorly suitable \\
\hline Large number of clients & $\begin{array}{c}\text { any architecture with a proxy } \\
\text { OSCAR, MQTT-SN }\end{array}$ & ACE DCAF, ACE OAuth & $\begin{array}{l}\text { architectures without proxy } \\
\text { DTLS, IPsec, OSCOAP }\end{array}$ \\
\hline High request frequency & $\begin{array}{c}\text { any architecture with a proxy } \\
\text { OSCAR, MQTT-SN }\end{array}$ & ACE DCAF, ACE OAuth & $\begin{array}{l}\text { architectures without proxy } \\
\text { DTLS, IPsec, OSCOAP }\end{array}$ \\
\hline $\begin{array}{l}\text { Deployments without a } \\
\text { dedicated authentication server }\end{array}$ & DTLS, IPsec & OSCOAP (if prior agreement) & any other architecture \\
\hline Authorization granularity & ACE DCAF, ACE OAuth, MQTT-SN & OSCAR & DTLS, IPsec, OSCOAP \\
\hline Deployments with actuators & - & any architecture except MQTT-SN & MQTT-SN \\
\hline Heterogeneous network & $\begin{array}{l}\text { architectures using negotiation protocols } \\
\text { ACE DCAF, MQTT-SN, DTLS, IPsec }\end{array}$ & - & $\begin{array}{c}\text { MQTT-SN } \\
\text { (nonnegotiable resource representations) }\end{array}$ \\
\hline Real-time clocks & ACE DCAF, ACE OAuth & any architecture except OSCAR & OSCAR \\
\hline Memory & MQTT-SN, ACE OAuth, ACE DCAF & OSCAR & $\begin{array}{l}\text { architectures without a dedicated device at } \\
\text { least for authentication: DTLS, IPsec, } \\
\text { OSCOAP (if great number of clients) }\end{array}$ \\
\hline Computation and batteries & MQTT-SN, ACE DCAF, ACE OAuth & DTLS, IPsec, OSCOAP & OSCAR \\
\hline
\end{tabular}

Table 16: Summary of the suitability of the architectures given specific constraints.

\subsection{Discussion}

Note for all architectures: none of them is providing a built-in mechanism for resource discovery, this should be performed by a combination of 6LoWPAN and CoAP (and DTLS for a secure discovery).

The principle of separation of the authentication process as used in Kerberos is useful to build a single sign on (SSO) authentication system for traditional enterprise networks with multiple applications (printers, servers, desktops, etc). Even if Kerberos is unsuitable for constrained environments, this can be used in another context, such as a privacy-aware deployment. For instance, a domain can trust an authentication entity from another domain, which is a trusted third party providing tickets to authenticated clients. An authorization entity could complete its task without knowing the client's identity using the ticket (with a signature or an authenticated code) from this third party proving that the client has been identified. The domain with the authentication entity has the client's identity, the other one does not require it. Also, useful information can be added in the ticket by the third party for further processing: clients may be categorized into groups, and this information is shared from the third party (authentication entity) to the authorization entity via the ticket. As a practical example, a printing company may authorize access to anyone authenticated by an authentication server from an associated enterprise, without knowing the identity of the end-user. The same is true for the DCAF architecture with its authentication servers. 


\section{Related work}

Security architectures are the subject of several documents from the International Telecommunication Union (ITU) such as [16], [18] and [17]. They define many concepts about security that are nowadays widespread in the literature, such as basic security services over-the-wire (authentication, access control, data confidentiality, data integrity and non-repudiation), security domain, authority and policy. These concepts are the basics of security in general and it is useful to share the same language but these documents do not attempt to provide any kind of implementation specifications. The ITU X8xx documents represent an abstract view on security architectures and their functionalities, but they are not suited to constrained environments and do not provide a concise, simple and intelligible definition of security architectures. These documents textually define concepts used in security architectures and basic security services. However, to our knowledge, no formal definition of security architectures has been described in the literature.

An effort is made to document most aspects of securing constrained environments networks by publishing few internet drafts such as security considerations [31], practical considerations along with implementation experiences [45] or secure bootstrapping [36].

Along with COSE message format, Cryptographic Message Syntax (CMS) is a standard notation and representation for cryptographic messages. CMS is used to digitally sign, digest, authenticate, or encrypt arbitrary message content, and uses the ASN.1 format that limits its use in constrained environments because of the message size.

Extended Authentication Protocol (EAP) is a framework designed to provide authentication protocol negotiation. Dozens of authentication protocols are available, derived from already known protocols such as TLS or IKEv2, to specific EAP authentication protocols to respond to particular constraints such as authentication from a phone. The study of all these protocols and their usage in a security architecture in a constrained environment would require a dedicated paper.

\section{Conclusion and future work}

In this paper we have described consistent solutions to security problems known as security architectures. A definition of this term has been established to lead to a better understanding of the solutions, see above. An overview of the main available security architectures was presented, and a comparison was made based on two scenarios with different deployment constraints.

Each architecture was summarized by their entities (functional components comprising it), situated in different domains, and by a set of relations (interactions between entities) describing the protocols used, communication properties (required or induced) and shared informations.

The comparison showed that none of the solutions met all the criterion, from the security or the constraint point of view. The different deployments and their constraints showed that none of the architectures performed well in every situation. However, a built-in proxy feature and cryptographic agility are key components to build efficient architectures. Future work may define a new security architecture matching the important properties found in this paper.

Secure group communication is still a difficult task, new architectures may include this network feature into account.

\section{References}

[1] Aragon, S., Tiloca, M., Raza, S., 2017. IPsec profile of ACE. Internet-Draft draft-aragon-ace-ipsec-profile-01. IETF Secretariat. URL: http: //www.ietf .org/internet-drafts/draft-aragon-ace-ipsec-profile-01.txt http://www.ietf .org/internet-drafts/ draft-aragon-ace-ipsec-profile-01.txt

[2] Barker, E., 2016. Recommendation for key management - part 1: General (revised), in: NIST Special Publication 800-57 Part 1, revision 4.

[3] Barnes, R., 2014. Use Cases and Requirements for JSON Object Signing and Encryption (JOSE). RFC 7165 (Informational). URL: http://www.ietf.org/rfc/rfc7165.txt

[4] Bellovin, S., Housley, R., 2005. Guidelines for Cryptographic Key Management. RFC 4107 (Best Current Practice). URL: http://www. ietf.org/rfc/rfc4107.txt

[5] Bormann, C., 2014. 6LoWPAN-GHC: Generic Header Compression for IPv6 over Low-Power Wireless Personal Area Networks (6LoWPANs). RFC 7400 (Proposed Standard). URL: http://www . ietf .org/rfc/rfc7400.txt

[6] Bormann, C., Ersue, M., Keranen, A., 2014. Terminology for Constrained-Node Networks. RFC 7228 (Informational). URL: http: //www.ietf.org/rfc/rfc7228.txt 
[7] Bormann, C., Hoffman, P., 2013. Concise Binary Object Representation (CBOR). RFC 7049 (Proposed Standard). URL: http://www . ietf.org/rfc/rfc7049.txt

[8] Callas, J., Donnerhacke, L., Finney, H., Shaw, D., Thayer, R., 2007. OpenPGP Message Format. RFC 4880 (Proposed Standard). URL: http://www.ietf.org/rfc/rfc4880.txt

[9] Stefanie Gerdes, Olaf Bergmann, Carsten Bormann, Goeran Selander, Ludwig Seitz, 2017. Datagram Transport Layer Security (DTLS) Profiles for Authentication and Authorization for Constrained Environments (ACE). Internet-Draft draft-ietf-ace-dtls-authorize-02. IETF Secretariat. URL: http://www.ietf.org/internet-drafts/draft-ietf-ace-dtls-authorize-02.txt http://www.ietf.org/ internet-drafts/draft-ietf-ace-dtls-authorize-02.txt

[10] Gerdes, S., Bergmann, O., Bormann, C., 2015. Delegated CoAP Authentication and Authorization Framework (DCAF). Working Draft.

[11] Granjal, J., Monteiro, E., Silva, J.S., 2015. Security for the Internet of Things: A Survey of Existing Protocols and Open Research Issues. IEEE Communications Surveys Tutorials 17, 1294-1312. doi 10.1109/COMST. 2015.2388550

[12] Granjal, J., Silva, R., Monteiro, E., Sa Silva, J., Boavida, F., 2008. Why is IPsec a Viable Option for Wireless Sensor Networks, in: 2008 5th IEEE International Conference on Mobile Ad Hoc and Sensor Systems, pp. 802-807. doi 10.1109/MAHSS . 2008.4660130

[13] Housley, R., 2015. Guidelines for Cryptographic Algorithm Agility and Selecting Mandatory-to-Implement Algorithms. RFC 7696 (Best Current Practice). URL: https://www.rfc-editor.org/rfc/rfc7696.txt doi 10.17487/RFC7696

[14] IEEE, 2006. IEEE 802.15.4 datasheet. Http://standards.ieee.org/getieee802/download/802.15.4-2006.pdf.

[15] Igoe, K., Solinas, J., 2009. AES Galois Counter Mode for the Secure Shell Transport Layer Protocol. RFC 5647 (Informational). URL: http://www.ietf.org/rfc/rfc5647.txt

[16] ITU, 1991. X.800 : Security Architecture for Open Systems Interconnection for CCITT Applications. URL: http://www.itu.int/rec/ T-REC-X.800-199103-I

[17] ITU, 1995. X.810 : Information Technology - Open Systems Interconnection - Security Frameworks for Open Systems: Overview.

[18] ITU, 2003. X.805 : Security Architecture for Systems Providing End-to-End Communications. URL: http://www.itu.int/rec/ T-REC-X.805-200310-I/en

[19] Jankiewicz, E., Loughney, J., Narten, T., 2011. IPv6 Node Requirements. RFC 6434 (Informational). URL: http: //www .ietf . org/rf c/ rfc6434.txt

[20] Kaufman, C., Hoffman, P., Nir, Y., Eronen, P., Kivinen, T., 2014. Internet Key Exchange Protocol Version 2 (IKEv2). URL: http: //www.ietf.org/rfc/rfc7296.txt

[21] Kent, S., 2005a. IP Authentication Header. RFC 4302 (Proposed Standard). URL: http://www.ietf .org/rfc/rfc4302.txt

[22] Kent, S., 2005b. IP Encapsulating Security Payload (ESP). RFC 4303 (Proposed Standard). URL: http: //www . ietf . org/rfc/rfc4303. txt

[23] Kent, S., Seo, K., 2005. Security Architecture for the Internet Protocol. RFC 4301 (Proposed Standard). URL: http://www.ietf .org/ rfc/rfc4301.txt

[24] Keoh, S.L., Kumar, S.S., Tschofenig, H., 2014. Securing the Internet of Things: A Standardization Perspective. IEEE Internet of Things Journal 1, 265-275. doi 10.1109/JIOT.2014.2323395

[25] Langley, A., Hamburg, M., Turner, S., 2016. Elliptic Curves for Security. RFC 7748 (Informational). URL: http://www .ietf .org/rfc/ rfc7748.txt

[26] Liu, A., Ning, P., 2008. TinyECC: A Configurable Library for Elliptic Curve Cryptography in Wireless Sensor Networks, in: Proceedings of the 7th International Conference on Information Processing in Sensor Networks, IEEE Computer Society, Washington, DC, USA. pp. 245-256. URL: http://dx.doi.org/10.1109/IPSN.2008.47 doi 10.1109/IPSN.2008.47

[27] Lopez, R., Garcia, D., 2017. EAP-based Authentication Service for CoAP. Internet-Draft draft-marin-ace-wg-coap-eap-06. IETF Secretariat. URL:http://www.ietf.org/internet-drafts/draft-marin-ace-wg-coap-eap-06.txt http://www.ietf.org/ internet-drafts/draft-marin-ace-wg-coap-eap-06.txt

[28] John Mattsson, 2017. Using Transport Layer Security (TLS) to Secure OSCORE. Internet-Draft draft-mattsson-ace-tls-oscore-00. IETF Secretariat. URL: http://www.ietf.org/internet-drafts/draft-mattsson-ace-tls-oscore-00.txt http://www.ietf.org/ internet-drafts/draft-mattsson-ace-tls-oscore-00.txt

[29] McGrew, D., Hoffman, P., 2014. Cryptographic Algorithm Implementation Requirements and Usage Guidance for Encapsulating Security Payload (ESP) and Authentication Header (AH). RFC 7321 (Proposed Standard). URL: http://www.ietf .org/rfc/rfc7321.txt

[30] Montenegro, G., Kushalnagar, N., Hui, J., Culler, D., 2007. Transmission of IPv6 Packets over IEEE 802.15.4 Networks. RFC 4944 (Proposed Standard). URL: http://www.ietf .org/rfc/rfc4944.txt

[31] Oscar Garcia Morchon, Sandeep Kumar, Mohit Sethi, 2018. State-of-the-Art and Challenges for the Internet of Things Security. Internet-Draft draft-irtf-t2trg-iot-seccons-14. IETF Secretariat. URL: http://www.ietf.org/internet-drafts/ draft-irtf-t2trg-iot-seccons-14.txt http://www.ietf.org/internet-drafts/draft-irtf-t2trg-iot-seccons-14. txt

[32] Neuman, C., Yu, T., Hartman, S., Raeburn, K., 2005. The Kerberos Network Authentication Service (V5). RFC 4120 (Proposed Standard). URL: http://www.ietf.org/rfc/rfc4120.txt

[33] OASIS, 2015. Message Queuing Telemetry Transport (MQTT). URL: https://docs.oasis-open.org/mqtt/mqtt/v3.1.1/ errata01/os/mqtt-v3.1.1-errata01-os-complete.html

[34] Rahman, A., Dijk, E., 2014. Group Communication for the Constrained Application Protocol (CoAP). RFC 7390 (Experimental). URL: http://www.ietf .org/rfc/rfc7390.txt

[35] Rescorla, E., Modadugu, N., 2012. Datagram Transport Layer Security Version 1.2. RFC 6347 (Proposed Standard). URL: http://www. ietf.org/rfc/rfc6347.txt

[36] Sarikaya, B., Sethi, M., Sangi, A., 2017. Secure IoT Bootstrapping: A Survey. Internet-Draft draft-sarikaya-t2trg-sbootstrapping-03. IETF Secretariat. URL: http://www.ietf.org/internet-drafts/draft-sarikaya-t2trg-sbootstrapping-03.txt http://www . ietf.org/internet-drafts/draft-sarikaya-t2trg-sbootstrapping-03.txt

[37] Schaad, J., 2017. CBOR Object Signing and Encryption (COSE). RFC 8152 (Proposed Standard). URL: https ://www.rf c-editor . org/ 
rfc/rfc8152.txt doi $10.17487 /$ RFC8152

[38] Schiller, J., 2005. Cryptographic Algorithms for Use in the Internet Key Exchange Version 2 (IKEv2). RFC 4307 (Proposed Standard). URL: http://www.ietf.org/rfc/rfc4307.txt

[39] Ludwig Seitz, Francesca Palombini, Martin Gunnarsson, Goeran Selander, 2018. OSCORE profile of the Authentication and Authorization for Constrained Environments Framework. Internet-Draft draft-ietf-ace-oscore-profile-01. IETF Secretariat. URL: http: //www.ietf.org/internet-drafts/draft-ietf-ace-oscore-profile-01.txt http://www.ietf.org/internet-drafts/ draft-ietf-ace-oscore-profile-01.txt

[40] Ludwig Seitz, Goeran Selander, Erik Wahlstroem, Samuel Erdtman, Hannes Tschofenig, 2017. Authentication and Authorization for Constrained Environments (ACE). Internet-Draft draft-ietf-ace-oauth-authz-09. IETF Secretariat. URL: http: //www.ietf.org/internet-drafts/draft-ietf-ace-oauth-authz-09.txt http://www.ietf.org/internet-drafts/ draft-ietf-ace-oauth-authz-09.txt

[41] Seitz, L., Gerdes, S., Selander, G., Mani, M., Kumar, S., 2016. Use Cases for Authentication and Authorization in Constrained Environments. RFC 7744 (Informational). URL: http://www. ietf.org/rfc/rfc7744.txt

[42] Goeran Selander, John Mattsson, Francesca Palombini, 2018. \begin{tabular}{|l|} 
HOC). Internet-Draft draft-selander-ace-cose-ecdhe-08. \\
internet-drafts/draft-selander-ace-cose-ecdhe-08.txt
\end{tabular} IETF Secretariat.

Ephemeral Diffie-Hellman Over COSE (EDdraft-selander-ace-cose-ecdhe-08.txt

[43] Selander, G., Mattsson, J., Palombini, F., Seitz, L., 2018. Object Security for Constrained RESTful Environments (OSCORE). Internet-Draft draft-ietf-core-object-security-12. IETF Secretariat. URL: http://www.ietf. org/internet-drafts/draft-ietf-core-object-security-12.txt http://www.ietf.org/internet-drafts/ draft-ietf-core-object-security-12.txt

[44] Cigdem Sengul, Anthony Kirby, Paul Fremantle, 2018. MQTT-TLS profile of ACE. Internet-Draft draft-sengul-ace-mqtt-tls-profile02. IETF Secretariat. URL: http://www.ietf.org/internet-drafts/draft-sengul-ace-mqtt-tls-profile-02.txt http: //www.ietf.org/internet-drafts/draft-sengul-ace-mqtt-tls-profile-02.txt

[45] Mohit Sethi, Jari Arkko, Ari Keranen, Heidi-Maria Back, 2018. Practical Considerations and Implementation Experiences in Securing Smart Object Networks. Internet-Draft draft-ietf-lwig-crypto-sensors-06. IETF Secretariat. URL: http: //www.ietf.org/internet-drafts/draft-ietf-lwig-crypto-sensors-06.txt http://www.ietf.org/internet-drafts/ draft-ietf-lwig-crypto-sensors-06.txt

[46] Shelby, Z., Hartke, K., Bormann, C., 2014. The Constrained Application Protocol (CoAP). RFC 7252 (Proposed Standard). URL: http: //www.ietf.org/rfc/rfc7252.txt

[47] Tschofenig, H., Fossati, T., 2016. Transport Layer Security (TLS) / Datagram Transport Layer Security (DTLS) Profiles for the Internet of Things. RFC 7925 (Proposed Standard). URL: http://www. ietf .org/rfc/rfc7925.txt

[48] Mališa Vučinić, Bernard Tourancheau, Franck Rousseau, Andrzej Duda, Laurent Damon, Roberto Guizzetti, 2015. OSCAR: Object Security Architecture for the Internet of Things. Ad Hoc Networks 32, 3 - 16. URL: http://www . sciencedirect.com/science/article/pii/ S1570870514003126 internet of Things security and privacy: design methods and optimization.

[49] Blake Wilson, S., Bolyard, N., Gupta, V., Hawk, C., Moeller, B., 2006. Elliptic Curve Cryptography (ECC) Cipher Suites for Transport Layer Security (TLS). RFC 4492 (Informational). URL: http://www. ietf.org/rfc/rfc4492.txt

[50] Ylonen, T., Lonvick, C., 2006a. The Secure Shell (SSH) Authentication Protocol. RFC 4252 (Proposed Standard). URL: http://www. ietf.org/rfc/rfc4252.txt

[51] Ylonen, T., Lonvick, C., 2006b. The Secure Shell (SSH) Connection Protocol. RFC 4254 (Proposed Standard). URL: http: //www . ietf . org/rfc/rfc4254.txt

[52] Ylonen, T., Lonvick, C., 2006c. The Secure Shell (SSH) Protocol Architecture. RFC 4251 (Proposed Standard). URL: http://www . ietf . org/rfc/rfc4251.txt

[53] Ylonen, T., Lonvick, C., 2006d. The Secure Shell (SSH) Transport Layer Protocol. RFC 4253 (Proposed Standard). URL: http://www. ietf.org/rfc/rfc4253.txt 